\title{
EchoGéo
}

\section{Ecotourisme et parcs naturels : innovations et contradictions sud-africaines, le cas de HIP (Hluhluwe-Imfolozi Park)}

Fabrice Folio et Anaëlle Derroisne

\author{
(2) OpenEdition \\ Journals \\ Édition électronique \\ URL : https://journals.openedition.org/echogeo/11769 \\ DOI : 10.4000/echogeo.11769 \\ ISSN : 1963-1197 \\ Éditeur \\ Pôle de recherche pour l'organisation et la diffusion de l'information géographique (CNRS UMR 8586) \\ Référence électronique \\ Fabrice Folio et Anaëlle Derroisne, «Ecotourisme et parcs naturels : innovations et contradictions sud- \\ africaines, le cas de HIP (Hluhluwe-Imfolozi Park) », EchoGéo [En ligne], 13 | 2010, mis en ligne le 03 \\ août 2021, consulté le 03 août 2021. URL : http://journals.openedition.org/echogeo/11769 ; DOI \\ https://doi.org/10.4000/echogeo.11769
}

Ce document a été généré automatiquement le 3 août 2021.

EchoGéo est mis à disposition selon les termes de la licence Creative Commons Attribution - Pas d'Utilisation Commerciale - Pas de Modification 4.0 International (CC BY-NC-ND) 


\title{
Ecotourisme et parcs naturels : innovations et contradictions sud- africaines, le cas de HIP (Hluhluwe- Imfolozi Park)
}

\author{
Fabrice Folio et Anaëlle Derroisne
}

1 L'aire protégée de Hluhluwe-Imfolozi Park (HIP) se situe dans la contrée du Zululand (province du KwaZulu-Natal) au nord-est de l'Afrique du Sud. Parmi les plus vieilles réserves de chasse d'Afrique australe, HIP fait aujourd'hui l'objet de projets écotouristiques innovants. Le Parc est le cadre d'initiatives pionnières et parfois ambitieuses, qu'il se fait fort de mettre en avant dans les domaines de la protection environnementale, de l'exploitation touristique ou de l'association communautaire. Ce dernier aspect revêt beaucoup d'importance dans un pays où le contentieux historique, associé aux espaces protégés, demeure prégnant. Le legs de l'apartheid consiste en effet en un zonage spatial au sein des aires à forte biodiversité (Guyot, 2004), sur lequel un développement cloisonné risque de perdurer. Or, appuyée en cela par les acteurs supranationaux, la participation des communautés légitime à présent des politiques étatiques résolument axées sur la conservation à des fins touristiques (Christie, Crompton, 2001). Qu'est ce qui fonde le succès touristique de HIP et quels sont ses défis hérités ? Le Parc parvient-il à conduire une réelle politique participative, à l'intérieur d'un espace naturel historique préservé et exploité ? Derrière les effets d'annonce, il est à craindre que l'équilibre écotouristique ne soit que difficilement atteint au vu du contexte donné. Au-delà de notre hypothèse liminaire, l'exemple de HIP soulève plus largement des interrogations originales, rassemblées autour du couple innovation contradiction. Le Parc ne génère-t-il pas de nouvelles ambiguïtés socio - territoriales, par delà les matérialisations mises en avant?

2 Cet article vise à esquisser la nature de la compatibilité entre les intérêts de la protection de la biodiversité, de la viabilité économique et du développement local au sein et autour d'un parc public animalier sud-africain. La réflexion porte sur l'équation 
écotouristique, segment de la durabilité touristique, elle-même déclinaison sectorielle du développement durable. Le texte s'ouvre sur un rappel conceptuel de l'écotourisme et ses enjeux inhérents. Il s'attelle ensuite à notre espace d'étude, dressant d'abord ce qui fonde les atouts de HIP, pour finir par tenter de mesurer sa réalité écotouristique.

\section{Tourisme durable et écotourisme en Afrique (du Sud) : héritages et défis}

3 Cette première partie entend poser les bases terminologiques de notre étude, à travers un rappel de la définition des concepts d'écotourisme et de tourisme durable. Elles seront précédées des entrées thématiques qui les résument en partie, la première liée au tourisme dans une vision économique, la seconde associée à la protection dans une logique environnementale. Le contexte sud-africain et parfois africain sera évoqué, dans le but de commencer à esquisser les héritages mais aussi les spécificités de notre espace d'étude.

\section{L'entrée touristique : l'Afrique du Sud, un acteur majeur et original}

4 A l'échelle du continent africain, ce ne sont que certaines parties du territoire qui, à l'heure actuelle, sont concernées par l'arrivée de visiteurs internationaux (Afrique du nord, du sud et à degré moindre Afrique orientale). Le tourisme n'est réellement développé que dans un nombre limité de pays. Quatre d'entre eux captent l'essentiel des flux en 2006 selon l'OMT : l'Egypte (8,6 millions de touristes), l'Afrique du Sud (8,3 millions), la Tunisie et le Maroc (6,5 millions). On peut encore mentionner le Kenya, le Botswana ou la Tanzanie, (lesquels se sont habilement placés sur le créneau paysager et animalier), mais ces deux polarités territoriales - nord et sud du continent - sont un premier élément à retenir.

L'Afrique du Sud se classe en 2005 au vingt-sixième rang mondial, devant la Suisse ou l'Irlande. Son développement touristique, dans un contexte post-apartheid, est assez spectaculaire, bien qu'il semble se tasser depuis quelques années. Le pays s'étale sur plus de 1,2 millions $/ \mathrm{km}^{2}$ et détient un potentiel touristique varié ${ }^{1}$. Alors que l'Afrique du Nord reçoit l'essentiel de ses touristes de l'extérieur, la nation arc-en-ciel génère d'importants flux en provenance des pays voisins (Lamy-Giner, Guebourg, 2005). Il s'agit de l'une de ses originalités, une autre étant que le tourisme domestique est aussi, en ce pays, depuis longtemps développé. Si la vieille tradition récréative des Boers et des Britanniques justifie ce dernier point, la place importante du tourisme étranger africain se comprend elle par le poids économique du pays : le tourisme affinitaire, axé sur la chalandise, est en effet élevé, les visiteurs ultramarins appréciant avant tout, pour leur part, ses attractions naturelles.

Comme le rappelle P. Matlou (2001), fort de sa multitude de paysages, de son abondance de vie sauvage et de sa variété florale, le tourisme axé sur la nature est devenu un des fers de lance de l'économie nationale. On sait qu'historiquement, le secteur des loisirs touristiques en Afrique est surtout lié à la politique coloniale, notamment au sein des parcs naturels britanniques (Ndebele, 1998). Ceux-ci ont apprivoisé - par la qualité et le confort de leurs lodges - l'environnement sauvage en vue de l'exploration ou safari. L'Afrique du Sud en représente aujourd'hui un excellent exemple, encore que ce 
segment ne semble pas concerner localement un public hyper - sélectif mais a tendance à davantage se démocratiser.

7 L'essor du tourisme sud-africain repose enfin sur un professionnalisme et des prestations de qualité, mais des défis perdurent. Le pays reste en proie à une insécurité véhiculée par les médias (encore que les répartitions spatiales du phénomène sont importantes, Gervais-Lambony, 2009). Les évènements xénophobes de 2008 ont pu ajouter à une image qui se dégrade sensiblement. Les faibles niveaux de formation et d'infrastructures en milieu rural sont aussi des défis à relever. La décision de la FIFA d'organiser ici la coupe du monde de Football, en 2010, pourrait relancer l'activité. Outre les retombées financières directes et la réalisation de nouvelles infrastructures, le produit «Afrique du Sud» (jouant sur un créneau stratégique cumulant à la fois le traditionnel et le développé) sera diffusé dans le monde entier. Une aubaine dont entendent évidemment tirer profit décideurs et entrepreneurs.

\section{L'entrée environnementale : l'Afrique du Sud entre héritage colonial et espace - laboratoire}

Historiquement, c'est au $19^{\mathrm{e}}$ siècle que la pratique de la chasse - utilitaire puis de loisir au sein des game reserves - s'étend en Afrique, particulièrement dans les colonies britanniques. Symptomatiques des colonies de peuplement, les premières réserves répondent à un zonage spatial, aux côtés des "réserves" indigènes et des terres concédées aux colons. Ainsi en Afrique du Sud, la préservation est utilisée en tant qu'instrument ségrégatif (" apartheid vert »), la sanctuarisation écologique servant en parallèle à en expulser les populations locales (Guyot, 2004). Elles porteront, en germe, un ressentiment autochtone à l'encontre de la protection naturelle, aujourd'hui encore lourd de conséquences (cf. supra).

9 A peu près $10 \%$ de l'Afrique subsaharienne sont actuellement consacrés aux aires protégées. La Zambie et la Tanzanie occupent le haut du pavé, alors que $6,2 \% \mathrm{du}$ territoire de l'Afrique du Sud sont concernés (l'Union Internationale pour la Conservation de la Nature - UICN - préconisant une couverture de $10 \%$ dans chaque pays). Plus largement, se distingue une "banane verte" allant du Cap à Nairobi, incluant Afrique du Sud, Namibie, Botswana, Zimbabwe, Zambie, Tanzanie, Ouganda et Kenya (Giraut, Guyot, Houssay-Holzschuch, 2004). L'Afrique du Sud compte 218 Parcs nationaux/provinciaux et réserves naturelles privées. Parmi ceux-ci, les plus visités sont ceux détenant une ressource faunistique, comme le Kruger ou le Pilanesberg au nord du pays. D'autres Parcs, moins renommés, attirent également les touristes (tel le Parc National du Karoo ou celui d'Addo). Les aires naturelles, sauvages et les parcs nationaux occupent $53 \%$ du total des aires protégées nationales (World Ressource Institute, 2003), ce qui correspond aux catégories 1 et 2 de la classification de l'UICN (aire protégée intégrale ou à finalité environnementale et récréative). Les 21 parcs nationaux sont gérés par le SANParks (South African National Parks, sauf l'ex-Greater St Lucia géré par le Wetland Authority). Ils coexistent avec quantité d'espaces protégés publiques provinciaux (c'est le cas de notre espace d'étude), ainsi qu'avec de coûteuses et prestigieuses réserves privées qui les jouxtent bien souvent.

10 Epousant un courant écologiste récent, l'expression à la mode, à l'heure actuelle en Afrique australe, est celle de parc transfrontalier ou " parc de la paix » (Guyot, 2006c, Belaidi 2008). L'Afrique, où est née la Peace Park Fondation à l'origine du concept, est aux 
avant-gardes du phénomène, appuyé par les acteurs de la conservation et les bailleurs de fonds internationaux. Destiné à promouvoir l'intégration régionale par une politique commune de conservation et de développement via le tourisme, il associe surtout des parcs préexistants sud-africains à ceux des Etats voisins, permettant au pays de s'ériger en véritable puissance internationale ${ }^{2}$. Sa finalité est environnementale (protection étendue, zone ouverte aux grandes migrations animales) et économique (création de parcs animaliers prestigieux au processus de privatisation rampant). Mais deux autres volets sont avancés : celui géopolitique - ce sont d'anciennes zones de défiance et/ou de guerre transformées en zones de coopération - et surtout le volet social, qui promeut l'intégration des communautés aux différents projets.

\section{La synthèse espérée et augmentée : tourisme durable, responsable, écotourisme...}

11 Pour nombre d'occidentaux, l'attrait de l'Afrique réside dans la nature sauvage, ses paysages et ses grands fauves (Adams, Macshane, 1996). C'est le "tourisme de vision » qui domine le potentiel exploité (illustrations 1a et 1b). Le safari (« voyage» en swahili) incarne un produit touristique prisé, l'appareil photographique et la caméra ayant succédé à l'arme à feu. Le Kenya (Masaï Mara), la Tanzanie (Serengeti) et l'Afrique du Sud (Kruger) ont fait de cette forme de tourisme une de leurs ressources phares ${ }^{3}$. Le tourisme de nature y devient donc une affaire lucrative. Encore faut-il que cette activité serve au plus grand nombre.

Illustration 1a - Addo Elephant Park dans l'Eastern Cape

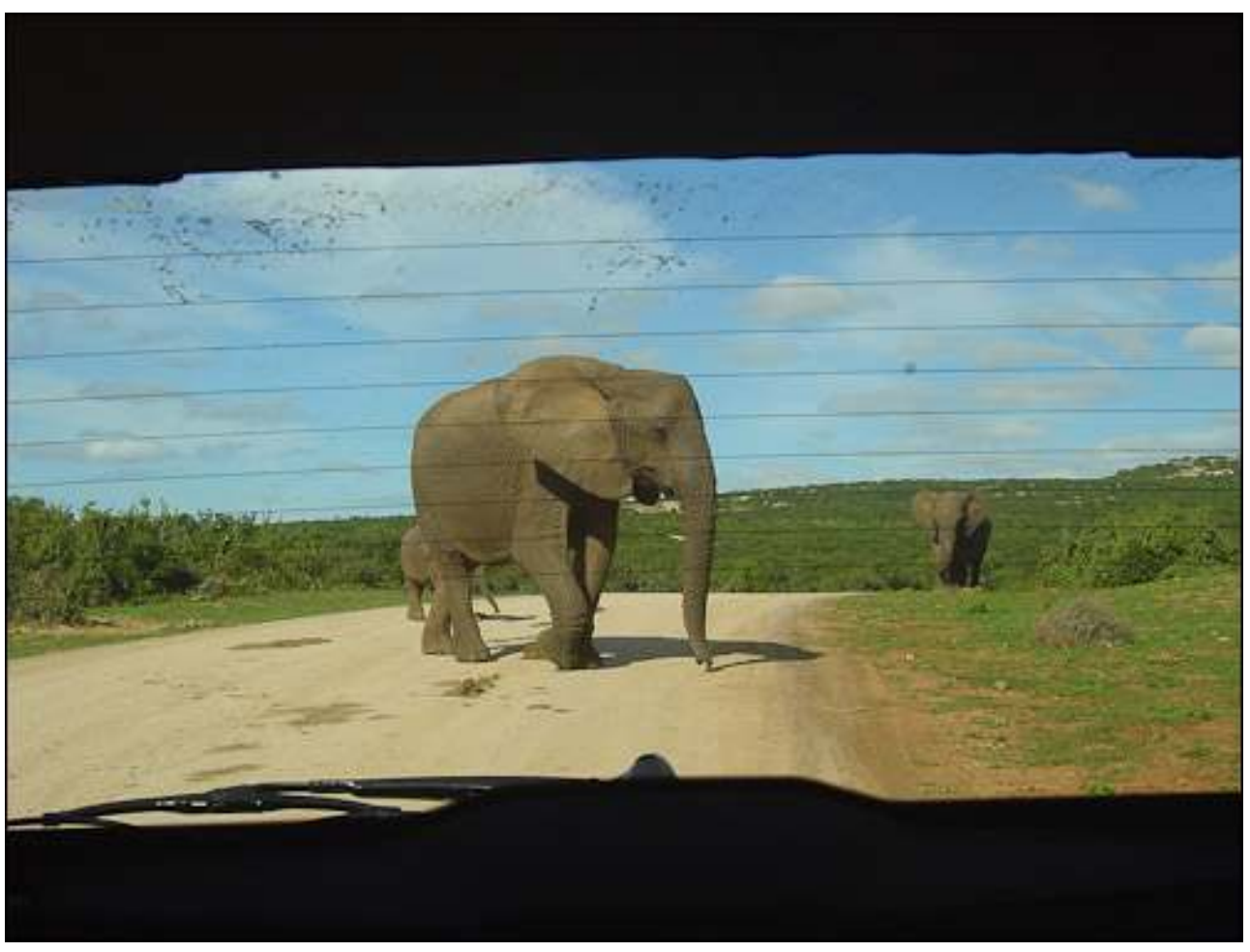




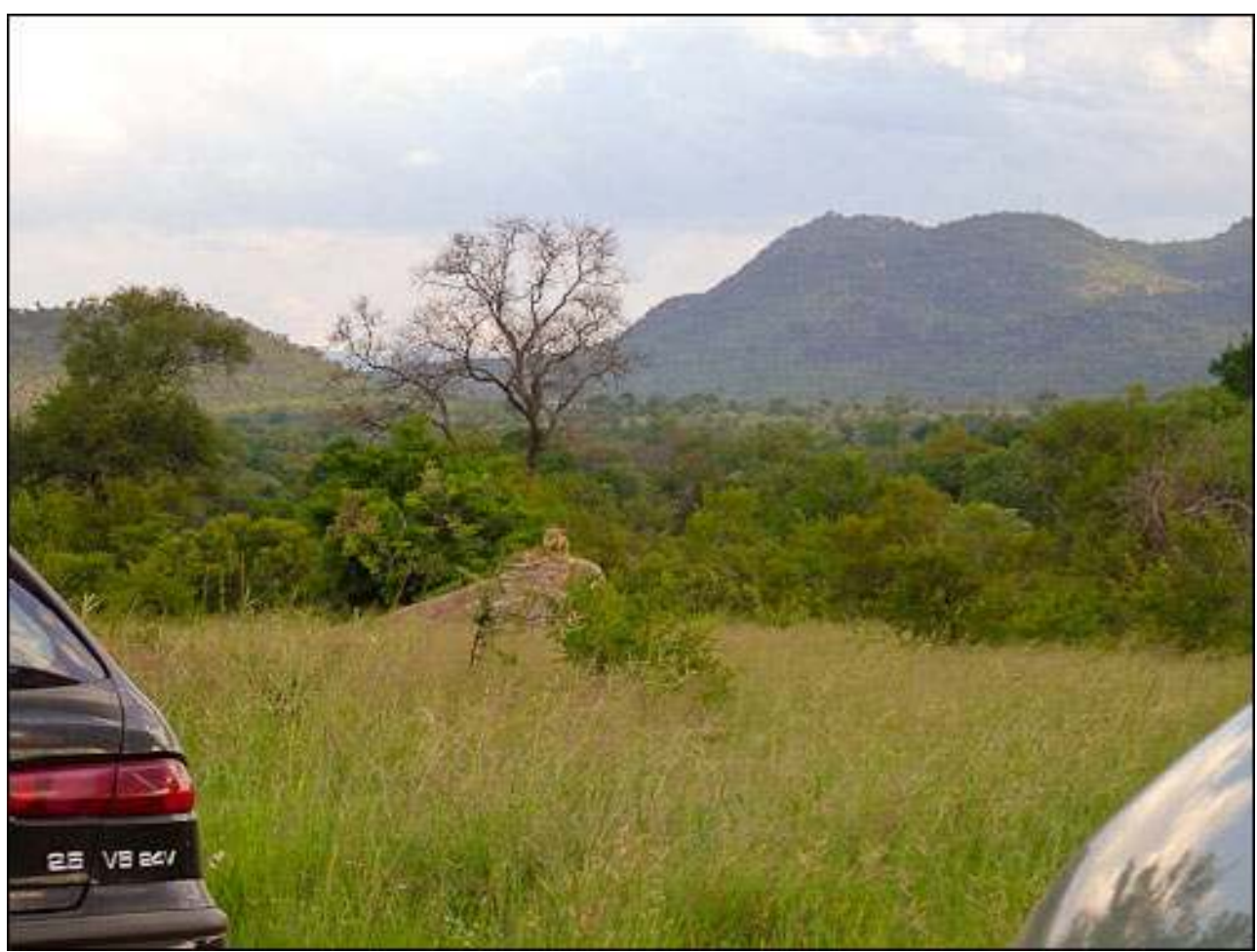

Les photographies 1a et 1b illustrent le tourisme de vision dans deux espaces fauniques sudafricains : le modèle national accorde une grande place à la motorisation individuelle sur de bonnes pistes, suscitant un contraste perceptif entre post-modernité et autonomie d'un côté, splendeur d'une nature séculaire menacée et menaçante de l'autre.

Auteur : Fabrice Folio.

En 1992, la conférence internationale de Rio a popularisé le développement durable, prônant la reconnaissance de l'environnement comme élément de développement actuel et futur 4 . A la suite de cette conférence, le tourisme est l'objet de réflexions destinées à définir de nouveaux concepts, dont celui du «tourisme durable». Ce dernier reprend le triptyque maintenant familier : «il doit être supportable à long terme sur le plan écologique, viable sur le plan économique et équitable sur le plan éthique et social pour les populations locales » (article 1, Charte du tourisme durable de l'OMT, adoptée en 1995). Dans l'idéal, le tourisme durable est capable de concilier l'essor économique, la protection naturelle et le bien-être des communautés (illustration 2). En Afrique du Sud, le terme voisin de «tourisme responsable » est privilégié par le Livre blanc sur le tourisme (1996) et sur l'environnement (1997). Il soutient une responsabilité positive et systémique des acteurs (touristes, gouvernement, entreprises, employeurs, populations locales) vis-à-vis d'un tourisme équilibré et durable, mentionnant notamment l'implication des communautés ou encore la sécurité des visiteurs. 


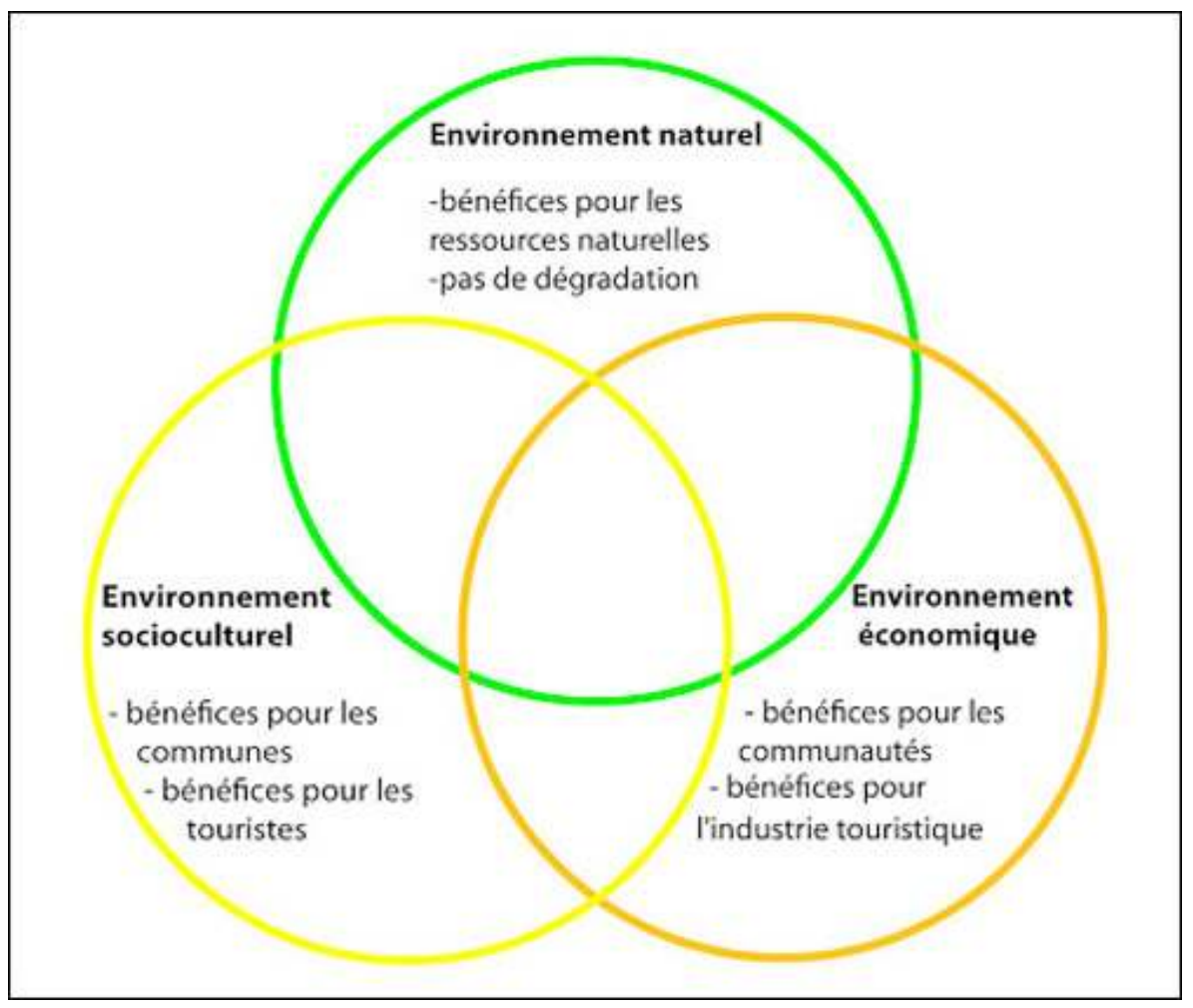

Source : d'après Wight, repris par Lubbe, 2003.

13 Parallèlement, un autre concept va émerger, ramené souvent à une forme de voyage responsable dans les espaces naturels: "l'écotourisme $»^{5}$. Si l'on se place sur la ligne des participants au premier Sommet mondial de l'écotourisme (Québec, 2002), l'écotourisme, en plus de contribuer à la protection du patrimoine naturel et culturel, s'affine en des principes :

- Il inclut les communautés locales et indigènes dans sa planification, son développement et son exploitation et contribue à leur bien-être ;

- Il propose aux visiteurs une interprétation du patrimoine naturel et culturel (s'ajoutant à tout ce qui concerne leur protection);

- Il se prête mieux à la pratique du voyage individuel ainsi qu'aux voyages organisés pour de petits groupes (OMT et PNUE, 2002).

De façon concise, R. K. Blamey (2001) amène à considérer trois dimensions qui constituent l'essence même de l'écotourisme : un tourisme axé sur la nature ; un besoin de durabilité; une composante éducative. La sensibilisation, les comportements respectueux envers la nature et les populations hôtes et enfin la contribution à leur développement sont la pierre angulaire de l'écotourisme. Ces stratégies sont préférables aux interdictions totales de visiteurs car, au final, le touriste, payeur, soutient en partie la conservation (Lubbe, 2003).

Pour B. Aylward et E. Lutz (2004), l'écotourisme peut faire profiter les aires protégées et les communautés locales de trois façons: en générant de l'argent pour gérer et protéger les habitats naturels et les espèces; en donnant l'opportunité aux populations de disposer de gains économiques grâce aux aires protégées; en offrant un moyen par lequel l'intérêt des individus - locaux et étrangers - pour la conservation peut être 
accru. C'est pour cette raison que la participation communautaire est activement prônée de nos jours, bien qu'elle puisse prendre plusieurs formes ${ }^{6}$ L'association des populations locales à l'activité des espaces protégés, adossée au rôle du secteur privé, est vue comme une nécessité face au défi patent de la pauvreté. En écho à P. Norton, consultant de l'OMT : «l'Afrique ne peut se permettre le luxe de créer des aires protégées au seul nom de la protection de la nature, tout simplement parce qu'elle compte trop de personnes qui ont faim » (2007). Celui-ci préconise une planification des aires protégées pour en faire la ressource centrale du développement durable par le tourisme. Qui plus est, la réintégration des acteurs locaux dans la protection s'impose comme une quasinécessité du fait que la conservation reste liée à un passé très autoritaire (Mackenzie, 1988). Pour autant, le segment écotouristique, loin de n'être qu'un tourisme axé sur la nature, reste dans les faits plutôt circonscrit. Le bilan des initiatives en Afrique australe demeure assez timide et est souvent réservé à une certaine élite (Koch, 2001, Guyot, 2004). On peut ici renvoyer à la contradiction établie par G. Rossi en 2000, quand au caractère finalement ambigu de l'écotourisme international, excluant le tourisme de masse pour pouvoir dégager des recettes sans impacter le milieu. HIP s'inscrit-il dans cette logique?

\section{Le parc animalier de Hluhluwe-Imfolozi : une biodiversité riche, un produit touristique ancien, accessible et de qualité}

Nous tenterons à présent de cerner, à travers un exemple sud-africain, l'adéquation entre ces aspects théoriques et la réalité du terrain. L'étude heuristique porte sur la réserve de Hluhluwe-Imfolozi (HIP) dans la partie septentrionale du KwaZulu-Natal (illustration 3$)^{7}$. Nous insisterons tout d'abord sur la singularité de cet espace faunique et plus largement sur ce qui a fait de lui un des hauts sites « à taille humaine » du pays (et ce malgré une géohistoire complexe) : une biodiversité de rang mondial associé à un produit touristique assez flexible. 
Illustration 3 - Localisation du Parc de Hluhluwe - Imfolozi

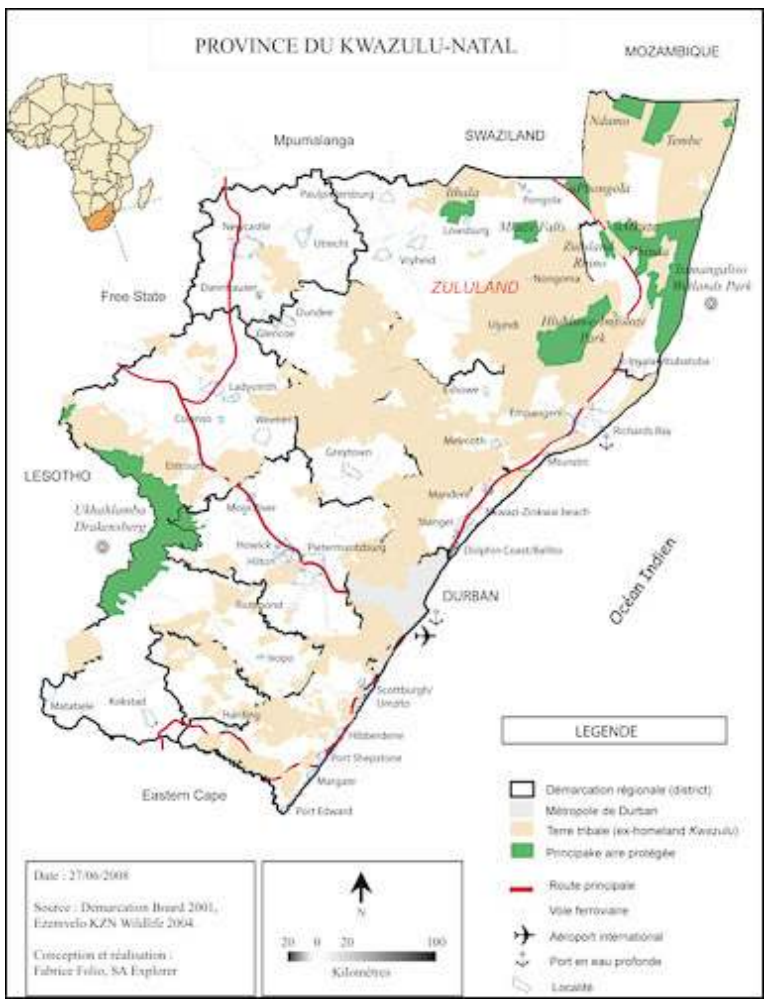

Source: Demarcation Board 2001, Ezemvelo KZN Wildlife 2004. Conception et réalisation : Fabrice Folio.

\section{Présentation de HIP : une Afrique naturelle des tableaux}

Les aires protégées représentent $8,4 \%$ de la province du KwaZulu-Natal, soit un taux supérieur aux $6,2 \%$ des terres nationales faisant l'objet d'une protection. Avec ses 96000 hectares, Hluhluwe-Imfolozi est la plus grande et l'une des plus anciennes surfaces protégées de cette province. Elle fait surtout figure de sanctuaire d'une biodiversité d'importance internationale. Grâce à son altitude et à sa position géographique, le Parc reçoit des précipitations plus abondantes que de nombreuses réserves sud-africaines ${ }^{8}$. Ces conditions climatiques sont à l'origine d'une végétation variée qui comprend des collines boisées, des forêts humides, denses ou à feuilles caduques, le bush caractéristique et les savanes... C'est le statut de protection dont l'espace a bénéficié qui a permis la politique de conservation d'une telle biodiversité. Outre les 1200 espèces de plantes recensées ${ }^{9}$, la diversité végétale a induit l'existence d'habitats exceptionnels pour une variété d'espèces animales (illustration 4) : fauves, oiseaux, amphibiens et reptiles. La réserve possède ainsi 84 espèces de mammifères et 350 espèces d'oiseaux pour un ensemble de plus de 60000 animaux (Charlton Perkins, 2002). HIP s'est surtout trouvé être le sanctuaire des rhinocéros, décimés ailleurs en Afrique. Le Parc détient respectivement $19 \%$ de la population mondiale de rhinocéros blancs et $17 \%$ des rhinocéros noirs (une espèce aujourd'hui en danger), tandis qu'il se permet d'approvisionner d'autres réserves. C'est en 1962 que « l'opération Rhino » fut lancée pour le sauvetage et la propagation de l'espèce. Confinés alors à Imfolozi où ils étaient menacés de surpopulation, une centaine de spécimens de rhinocéros blanc (plus lourd que le noir, à la tête plus large, broutant dans les plaines ouvertes) furent 
capturés et essaimés dans les réserves, parcs et zoos du monde entier. Aux côtés des grands de la brousse, les Big Fives - lion, éléphant, rhinocéros, buffle et léopard - aux stocks dûment surveillés (baguage, collier, puce électronique...), HIP héberge enfin une faune herbivore et carnivore diversifiée qui fait le bonheur du visiteur patient et attentif: girafes, zèbres, antilopes - Koudou, Nyala et l'omniprésent Impala -, phacochères, singes, hyènes sans oublier les vautours, serpents et autres crocodiles.

Illustration $4 a-$ HIP : paysage touristique

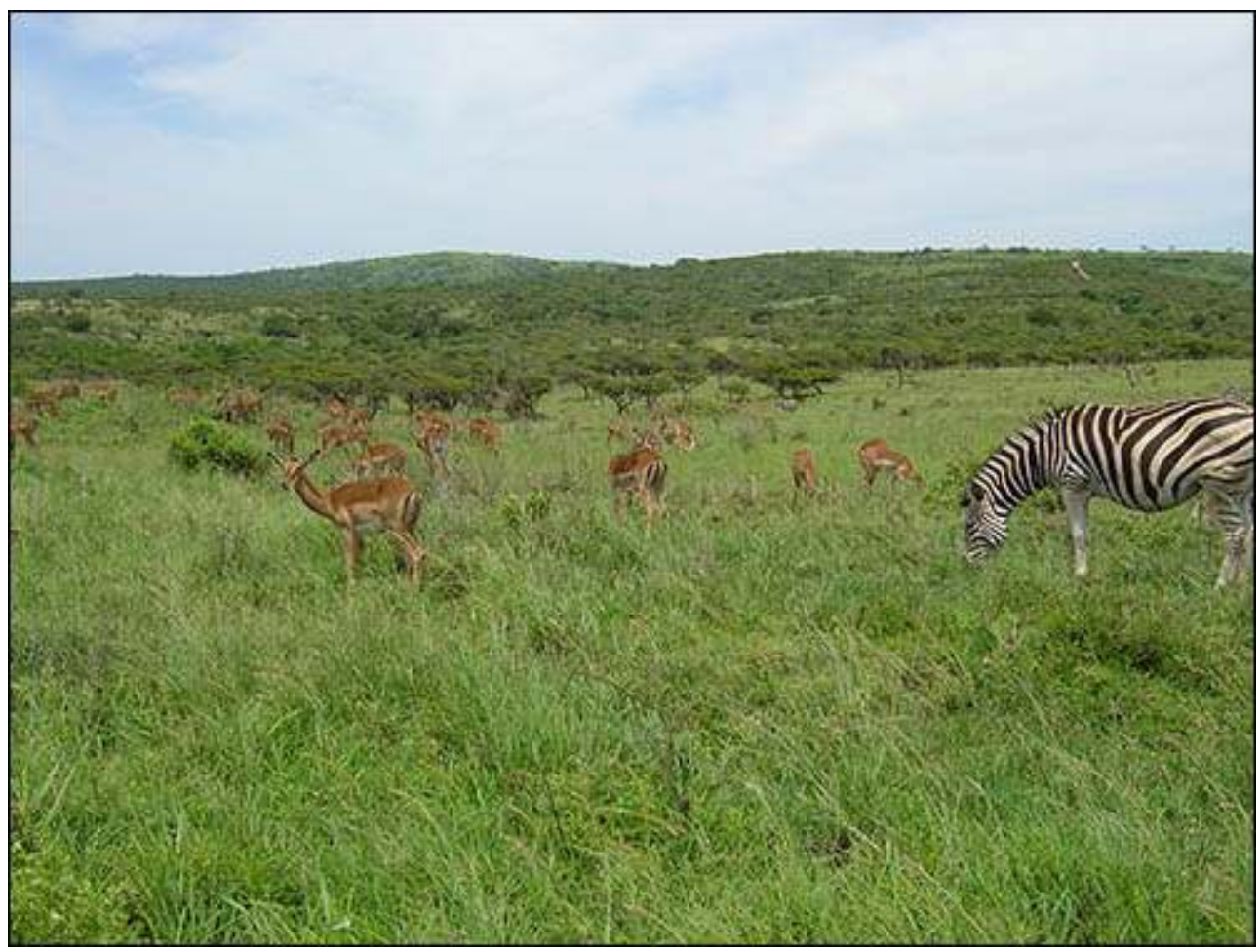




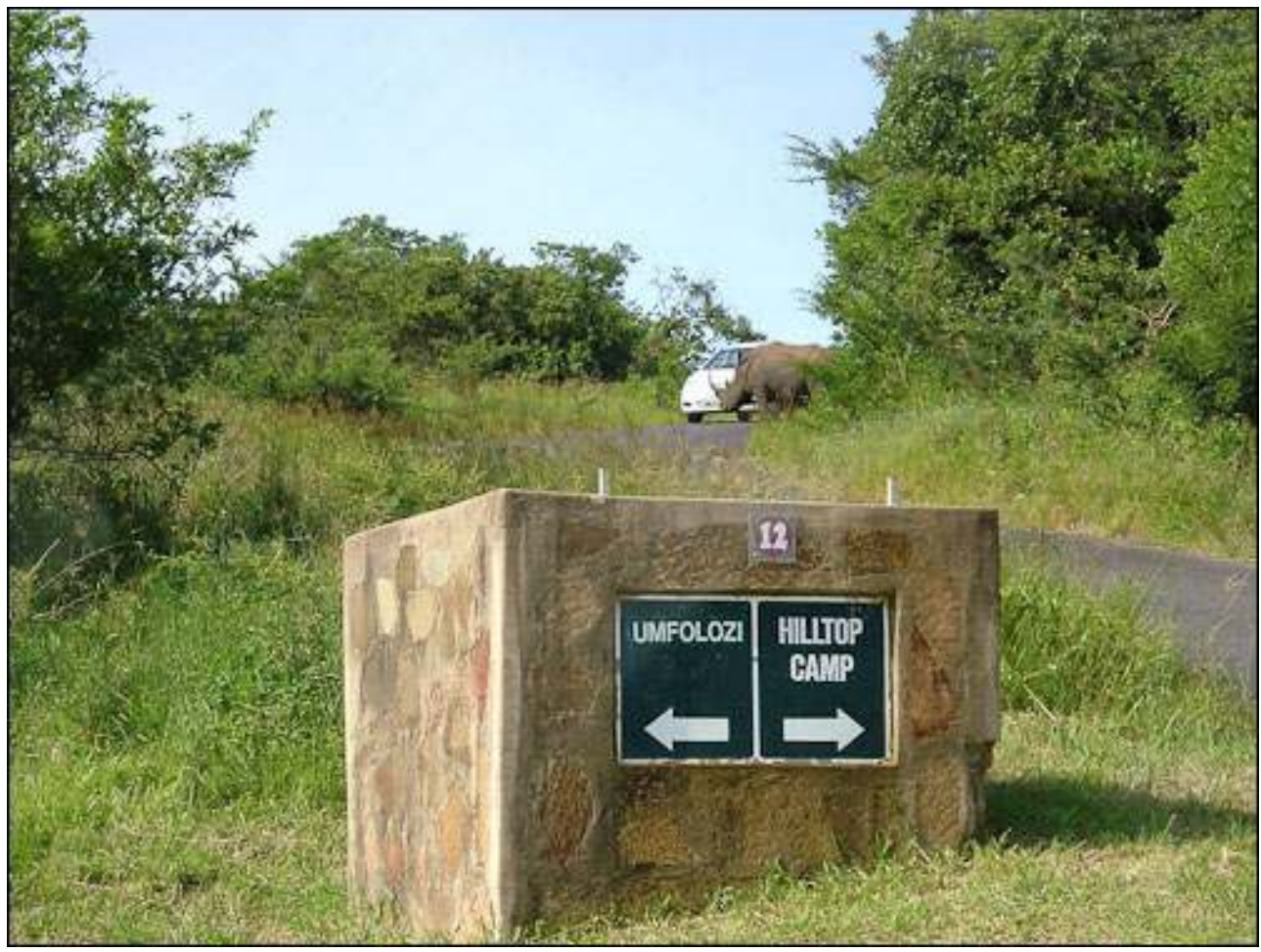

La présence d'une forte biodiversité dans un espace naturel vaste, constitue un bel atout pour l'activité touristique de HIP. Notons qu'aux côtés de possibilités de découverte pédestre et motorisée, bien encadrées, des sites d'observation cachés parsèment le parc (le plus souvent auprès des points d'eau). A l'exception de ces endroits désignés (auxquels on peut ajouter les aires de pique-nique et les camps), les visiteurs ne doivent sous aucun prétexte sortir de leur véhicule. Des attaques de rhinocéros ou d'éléphants se sont déjà déroulées, notamment lors de randonnées guidées (The Mercury, 2003-b, 2004).

Auteur : Fabrice Folio.

Les deux sections du Parc (Hluhluwe et Imfolozi), deux réserves initiales de différente taille, sont liées par un corridor, ponctué d'une route asphaltée reliant la localité de Nongoma dans l'intérieur à la côte. Avec 23067 hectares, la section nord de Hluhluwe est la plus petite en taille mais elle présente une réelle diversité paysagère (aidée en cela par la topographie et un complexe système de rivières). Ses points de vue en hauteur, ses berges touffues et ses plans d'eau (creusés naturellement ou artificiels), où s'abreuvent les animaux, comptent parmi ses points forts (en plus de sa richesse ornithologique). La végétation est toutefois davantage luxuriante, ce qui rend ardue la vision faunique en période estivale chaude et humide. Une route en dur la dessert, relayée par un réseau secondaire de voies gravillonnées (illustration 5). A Imfolozi, dans la vaste section située au sud du parc (47 753 hectares), le couvert végétatif est surtout composé de savanes arborées (le bushveld), dont les couleurs jaune et ocre en saison sèche, s'étendent à perte de vue. La section est desservie, comme sa voisine, par une route principale goudronnée qui laisse place, à partir d'une quinzaine de kilomètres, à un entrelacs de pistes en forme de boucles, auréolant la moitié nord-est de la section ; toute sa moitié sud n'est par ailleurs aucunement desservie, comme on le verra plus avant. 


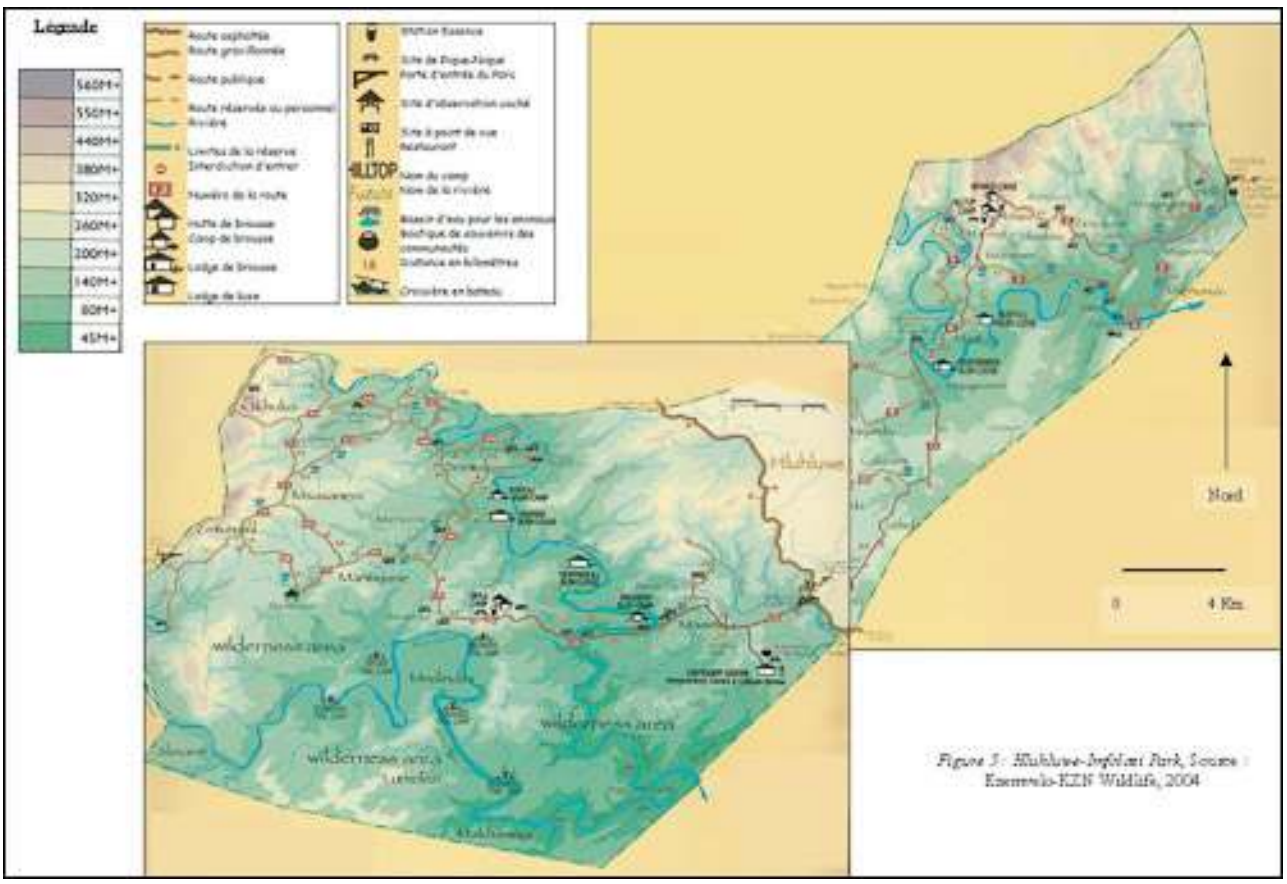

Source : Ezemvelo-KZN Wildlife, 2004.

\section{Rapports de force historiques et logiques d'acteurs : un espace complexe}

La date de 1895 marque la proclamation de cet espace en tant que première réserve d'animaux sauvages du Natal. Dans le détail, la géohistoire de cette aire « naturelle » en dit toutefois beaucoup sur les conflits d'acteurs pour l'occupation et l'utilisation des espaces à forte biodiversité. Elle complexifie aussi la somme des lieux communs ressassés au sujet des aires protégées en Afrique dite anglophone soit, chronologiquement: espace-ressource fusionnel pour les indigènes; délimitation coloniale et activité cynégétique de loisir ; idéaux de la conservation s'imposant et lutte contre le braconnage des Africains.

Il est par exemple utile de rappeler que les rois Zoulou Dingiswayo et Shaka avaient délimité, en cet endroit, un espace pour la chasse royale. Ce dernier, qui voulait aussi contenir au loin les tribus ennemies, fut l'instigateur de grandes traques d'animaux dans la région au début du $19^{\mathrm{e}}$ siècle. Des traces de ses fosses piégeuses attestent encore aujourd'hui de ces battues près de la confluence des rivières Black et White Mfolozi. A partir de 1840, les animaux subirent les assauts des "Grands chasseurs Blancs » qui arrivèrent par wagon de la colonie du Cap et de Port Natal (aujourd'hui la ville de Durban) : peaux de bêtes, ivoire, cornes de rhinocéros font alors partie du butin de chasse des Baldwin, Leslie et autres Drummond (qui paradoxalement alimentent de nombreux musées et contribuent à un inventaire de la faune). Après la guerre anglozouloue (1879), les autorités mettent en défens l'espace et déclarent formellement les Hluhluwe Valley reserve et Umfolozi Junction Reserve sanctuaires de chasse d'animaux sauvages en 1895. Elles sont d'abord gérées par l'administration britannique du Zululand puis par la colonie du Natal à partir de 1897. L'apparition de ces réserves est 
motivée par un premier élan conservationniste (devant la diminution du stock d'animaux), tout en concédant que les peuples autochtones ne peuvent s'approprier ces sites naturels grandioses. Alors qu'au départ, l'administration du Natal tolère la présence communautaire dans l'enceinte des réserves, elle l'y exclut dans les années 1930, forgeant ainsi un espace naturel à fréquentation élective.

Les réserves vont par la suite alterner des épisodes de conservation et de lutte contre le braconnage (à l'initiative de personnages notoires tels que I. Player ou H.B. Potter) et une logique de réduction/enfermement (Brooks, 2001) et d'ouverture à la chasse "sanitaire », sous la pression des agriculteurs. A partir de 1916, des zones tampons (autour de Hluhluwe et aux abords ouest et sud de Imfolozi) sont créées séparant réserves et terres agricoles. Dès 1919, s'ouvre une période tumultueuse avec les fermiers blancs voisins qui mettent en avant le danger de la nagana ; épizootie portée notamment par les buffles et transmise par la mouche tsé-tsé, elle décime le bétail domestique. Après une campagne active (qui voit l'expropriation du clan Manklakazi de la zone du corridor pour motif sanitaire et la disparition des zèbres à Imfolozi), la trypanosomiase animale ralentit dans les années 50 , sous le coup d'épandage massif de pesticides (DDT) et surtout des pièges de filet de R.H.T.P. Harris.

En 1947, l'ensemble des réserves du Zululand tombe sous la responsabilité du Natal Parks Board - NPB - et non plus du National Parks Board créé en 1926. Cela marque l'ancrage d'une identité provinciale anglo-saxonne dans la préservation. Des cottages attirent les visiteurs à Hluhluwe en 1935 , tandis que les premières huttes sont ouvertes au camp de Mpila en 1952 à Imfolozi. Des barrières sont établies en 1964 ; aussitôt, des négociations sont menées entre équipe du Parc et chefs locaux pour contrer le braconnage, tandis que des espèces animales (girafe, guépard, éléphant en 1981) sont réintroduites. La coalescence des réserves et du corridor verra le jour en 1989 pour une libre circulation de la faune.

Un nouveau rapport de force politique s'instaure sous l'apartheid. Le Parc se trouve enserré de terres tribales sous la juridiction du bantoustan Kwazulu, dans le cadre du "grand apartheid» (bien qu'il reste géré par le NPB). Face au Natal Parks Board, dont l'attitude envers les Noirs est critiquée (interventionnisme écologique), le Kwazulu Bureau of Natural Ressources est instauré pour gérer les réserves naturelles placées sur le Kwazulu. L'organisme entend accorder une plus grande place aux populations riveraines dans un souci de conservation. Gérant alors des réserves situées surtout dans le nord de la province (principalement au Maputaland: Ndumo etc.), son rôle sera équivoque: appuyé par le régime d'apartheid dans son objectif d'affaiblir l'African National Congress et de renforcer l'Inkataha pro-zoulou, il sera soupçonné de verser dans le trafic d'ivoire avec le Mozambique et de servir de couverture à des unités paramilitaires (Aylward, Lutz, 1994). Mais doté de sa tutelle politique et réputé proche des édiles traditionnels, son intégration est vue comme une nécessité dans le cadre de la réconciliation démocratique. On lui concède aujourd'hui les balbutiements provinciaux d'une politique de redistribution locale; du NPB, l'histoire officielle va retenir l'élan touristique et surtout l'expertise dans la protection de la biodiversité, à défaut pour ce dernier d'avoir été l'acteur majeur de la pensée conservationniste coloniale (EKZNW, Celebrates 10 Years of Democracy, 2004). Depuis 2000, HIP est géré par le Ezemvelo KZN Wildlife (EKZNW), organisme en charge des 110 espaces protégés de la province réunifiée du KwaZulu-Natal. La structure, au toponyme anglais et zoulou et à l'effigie du rhinocéros et du bouclier zoulou, est née de la difficile fusion des deux 
agences passées. Pouvant disposer de ses finances, elle est responsable devant la législation provinciale et le Ministère de l'Environnement.

\section{L'activité touristique contemporaine : flexibilité et diversité relative des visiteurs}

Il est de nos jours aisé d'explorer la totalité du parc animalier de HIP et d'y distinguer la variété de ses paysages et biomes. L'activité de game viewing est très appréciée des visiteurs qui bénéficient de la mise en valeur du Parc et plus largement du savoir-faire hérité ; cela leur laisse le loisir d'évoluer assez librement dans la réserve et d'observer ses diverses espèces, souvent confinés dans leur véhicule. A HIP, les visiteurs peuvent parcourir $214 \mathrm{~km}$ de sentiers touristiques ou game viewing drive (illustrations 4 et 5). Ces sentiers peuvent se découvrir seul (auto trails), avec l'aide de brochures explicatives détaillant les points d'intérêt du Parc (qu'il s'agisse de géologie, de faune ou de flore). Ces livres d'information sont disponibles aux différentes entrées, dans les centres d'accueil touristiques extérieurs ou dans les camps d'hébergement. Des tours en véhicules ouverts de type $4 \mathrm{X} 4$ sont aussi organisés par le EKZNW, ainsi que par des prestataires privés (Insinkwe Safaris, Bib's Tours...) travaillant avec les clients des hôtels/ auberges de jeunesse extérieurs au Parc et parfois éloignés ${ }^{10}$. En parallèle, des randonnées guidées à l'intérieur de la forêt indigène, les wilderness trails, sont possibles à différentes saisons, notamment les plus fraîches (entre mars et fin novembre). Les visiteurs, au nombre maximum de 8 participants, sont accompagnés de deux rangers naturalistes de l'équipe du KZN Wildlife, expérimentés et armés. Ils ont la possibilité de choisir parmi différentes prestations soit, opter pour la randonnée de deux heures, ou encore le safari-photo/camping de plusieurs jours. Pour finir, des croisières en bateau, d'une capacité de 40 places, permettent de longer une portion des berges de Hluhluwe. Les possibilités de découverte sont par conséquent très variées.

Depuis le $1^{\text {er }}$ novembre 2003, les visiteurs de HIP ne paient plus l'entrée à proprement parler, mais d'une "taxe de conservation prélevée sur les droits d'entrée » ou community levy. Elle s'élève actuellement à 80 rands par personne et par jour (environ 7,5 euros). Les visiteurs sud-africains et membres de la SADC, qui peuvent justifier de leur identité, ne déboursent eux que 40 rands pour la visite du Parc. Seuls les visiteurs qui ont choisi d'être hébergés à l'intérieur de la réserve sont acquittés des droits d'entrée, désormais inclus. Il est possible de séjourner dans le Parc dans les espaces aménagés à cet effet. En termes de prestations locales, on oscille sur place entre les aires de safari-camping (tels Mpila) et les luxueux lodges - Hilltop Camp, Muntulu, Gqoyeni - disposant de services de qualité (du cuisinier au ranger avec parfois la piscine), sans oublier les camps rustiques installés dans le bush (Sontuli). HIP propose ainsi des produits adaptés à diverses clientèles, déclinant autant de degrés de confort. Les deux principaux centres d'accueil sont les camps HillTop et Mpila, situés respectivement dans les deux sections. A HillTop, le visiteur pourra, à titre d'exemple, trouver un restaurant panoramique, une boutique de souvenirs, une station essence et même un centre de conférences qui s'ajoutent aux informations du jour sur la localisation de la faune.

S'il n'est pas aisé de quantifier la fréquentation touristique du Parc, on sait qu'elle tend vers les 450000 visiteurs à l'année (EKZNW). Des questionnaires ont été conduits pour compléter l'analyse ${ }^{11}$. La présence de touristes étrangers ultramarins coexistant avec 
un fort contingent de touristes nationaux est une caractéristique originale qui renvoie à une réalité nationale (même si au final un profil socio-économique assez typé se démarque). A la lecture des résultats, le taux de satisfaction s'avère correct : $87 \%$ des sondés ont estimé être très satisfaits de leur visite à Hluhluwe-Imfolozi. Beaucoup d'entre eux ont expliqué qu'ils apprécient les paysages immenses et paisibles, la sensation de vivre une expérience intime avec la nature et la faune visible. Certes HIP est moins grand et peuplé que par exemple l'illustre Kruger Parc géré par le SANParks (1 900000 ha pour près de 200000 espèces animales). HIP ne représente que $5 \%$ de la taille du plus grand parc sud-africain. Mais si on n'y trouve que $68 \%$ des plantes présentes au Kruger, il recouvre parfois des espèces supérieures en nombre, comme les rhinocéros (7 000 à HIP contre 3000 au Kruger). De plus, ses possibilités de vision de la faune sont de haute tenue : quelques visiteurs ont confié que les espaces moins étendus mais tout aussi grandioses de HIP incitent moins à la dispersion que d'autres vastes réserves. Du fait d'une concentration des routes dans certaines zones du Parc - et non d'un quadrillage lâche -, ils ont pu accorder plus de temps à l'observation. Aussi, sontils parvenus à apercevoir autant et parfois même plus d'animaux à HIP qu'au Kruger et ce en moins de temps. Il a aussi particulièrement été apprécié le nombre sensiblement moins élevé de véhicules à HIP qu'au populaire Kruger, où la densité automobile peut rapidement irriter...

\section{L'écotourisme à HIP : innovations et contradictions socio - territoriales}

27 La réalité écotouristique d'un tel espace est intéressante à mesurer. En effet, comme cela sera vu en cette partie, les spécificités locales s'associent à un contexte national et continental pour faire de HIP un espace singulier, où les innovations majeures dans le domaine de l'écotourisme côtoient certaines contradictions socio - territoriales. Nous passerons ici en revue, d'abord les versants économiques et environnementaux du Parc, ensuite associatifs, avant d'ébaucher une réflexion de fond sur la teneur de la conservation locale ${ }^{12}$.

\section{Protection environnementale et viabilité économique : des engagements respectés}

Du point de vue de l'effectivité économique, sans atteindre le niveau d'autres réserves d'Afrique du Sud, HIP se positionne bien au plan national. Le Parc dégage des excédents (KZN Wildlife, 2001, The Mercury, 2003-a). Au total, ses revenus annuels s'élèvent à environ 30 millions de rand (2,8 millions d'euros) et ses dépenses à R17 millions (1,6 $\mathrm{M} €)$. Sa relative renommée, le Parc la doit à sa situation dans cette région sauvage et historique, qui compose le cœur du pays zoulou. La possibilité pour un touriste de composer un «tour» naturel et culturel dans le nord du KwaZulu-Natal, cumulant différents sites sur un nombre de jours défini, fait de lui une valeur sûre ${ }^{13}$. Les touristes privilégiant - pour des questions de budget - les réserves publiques dotées des Big Fives, tendent ainsi à visiter HIP, que leur vendent d'ailleurs les tours opérateurs de Durban ou de Richards Bay. Quant à l'accessibilité du Parc, elle est convenable avec une signalétique correcte. Trois entrées sont disponibles (Cengeni, Mémorial et Nyalazi Gates), dont celles à partir de la Nationale 2, longeant la côte de part et d'autre de Durban. La 
voie menant au Memorial Gate, une des entrées les plus arpentées, a été refaite et elle n'est ni saturée, ni ne contraste trop fortement avec le paysage environnant. A l'intérieur, la gamme des prestations et des hébergements de HIP est, on l'a vu, variée (du pavillon luxueux à la tente rudimentaire). Les formules de découverte sont modulées et HIP atteint, pour ses hébergements, un taux d'occupation annuel moyen de $75 \%$.

Il a été tenté d'intégrer au mieux les logements à l'environnement ${ }^{14}$. Aux alentours, les aires de pique-nique et les abris de la réserve sont construits dans des matériaux légers qui essayent de peu dénaturer l'écosystème. Notons également qu'un quota du nombre de voitures existe lors des pics de fréquentation touristique en haute saison (mois de décembre, janvier et juillet). Mais la véritable valeur ajoutée de HIP est ailleurs : à l'intérieur des 66000 hectares de la partie sud de Imfolozi, une large portion de terres équivalente à 30000 hectares a été laissée vierge de tout aménagement (illustration 5). Aucun véhicule n'est autorisé à pénétrer dans cet espace. C'est ici que le Parc propose son activité écotouristique remarquable : les randonnées guidées à pied ou à cheval (12 à $15 \mathrm{~km}$ ), en petits groupes, sur plusieurs jours, avec des rangers. Apparu à la fin des années 1950, ce concept, pionnier en Afrique du Sud, a depuis été apprécié par plus de 100000 personnes. L'objectif du EKZNW est donc de protéger l'environnement d'une sur-fréquentation dans un premier temps, tout en sensibilisant à une utilisation durable de cet héritage dans un second temps. De la sorte, il atteint son objectif de préservation et d'éducation. Enfin, dans le cadre de relations de bon voisinage avec les aires riveraines (cf. supra), des programmes de sensibilisation à la biodiversité ont été constitués (comme le Schools biodiversity education programme). HIP est par conséquent un espace naturel où la protection est réelle et la fréquentation surveillée ; en cela c'est une réserve exploitée, s'éloignant des réserves « de papier » ou " saturées » que pointe du doigt B. Calas (2003) en Afrique orientale anglophone. Le débat, à ce niveau, peut se déplacer sur le caractère interventionniste de la protection de ce milieu, présenté comme primitif. Le Parc fonctionne en effet comme un modèle réduit et fermé en territoire sauvage d'origine (Carron, 2005); la délimitation de son périmètre, le strict contrôle de la faune et de la flore pourraient de ce fait être caractérisés "d'ingérence écologique " (Rossi, 2000). C'est là tout le paradoxe de l'approche conservationniste dans des écosystèmes dynamiques mais menacés...

\section{Une politique associative des communautés locales mise en avant}

Dernier élément - trop souvent minoré - du tryptique de la durabilité (soit son versant " social »), les répercussions écotouristiques de HIP sur les populations voisines sont à présent à examiner de près, devant le potentiel de développement et de réconciliation qu'elles peuvent localement incarner. L'espace entourant HIP est constitué de villages plus ou moins structurés et d'un habitat diffus (mêlant hutte traditionnelle et bâti en dur), où règne la propriété collective (illustration 6). On compte dix autorités tribales bordières, dirigées par un chef coutumier : l'inKhosi. Ce serait plus de 700000 personnes qui vivent aux alentours du Parc.

31 HIP est tout d'abord un employeur dans la région avec un total de 268 postes subventionnés par l'Etat et de 136 postes associés au secteur touristique. Les engagements pris par le EKZNW favorisent le recrutement local. Aussi, dans le cas du HillTop camp à Hluhluwe, $91 \%$ des employés résident dans région. Uniquement par le 
biais des salaires, HIP contribuerait à hauteur de R7,5 millions $(0,7 \mathrm{M} €)$ dans l'économie environnante (cela, sans compter les emplois créés avec les entreprises de services ou de fourniture liées à la réserve). De surcroît, dans le cadre du Hluhluwe-Imfolozi Park Community Conservation Programme (qui associe les autorités traditionnelles à la conservation), un Park Neighbour Partnership Programme a été institué en 1992 afin d'encourager le développement local dans une logique partenariale. On peut citer quelques-unes des initiatives établies : à un kilomètre avant l'entrée de Hluhluwe, un jardin potager, Zizamela, est tenu par des membres villageois en vue de la consommation locale; les surplus sont vendus aux restaurants des camps situés dans les réserves. A l'intérieur du Parc, se trouvent aussi les Vulamehlo Craft Market et Vukuzame Craft Center : ils permettent aux individus d'améliorer leurs revenus par la vente d'artisanat local aux touristes. HIP se veut par ailleurs utile par la mise à disposition de ressources servant à la production d'énergie, à l'habitat ou à la médecine traditionnelle $(M u t h i)^{15}$. Cet accès est utile aux protagonistes puisqu'il assiste l'équipe du Parc dans le contrôle de l'empiètement du bush, tout en répondant à certains besoins des populations locales. Enfin, des opérations ponctuelles d'éradication manuelle de pestes végétales (comme la Chromolaena odorata qui met en péril l'habitat des rhinocéros) impliquent en priorité les sans-emplois de la région. On reste là dans une approche essentiellement associative.

Illustration 6 - Aire villageoise de Mkhwanazi

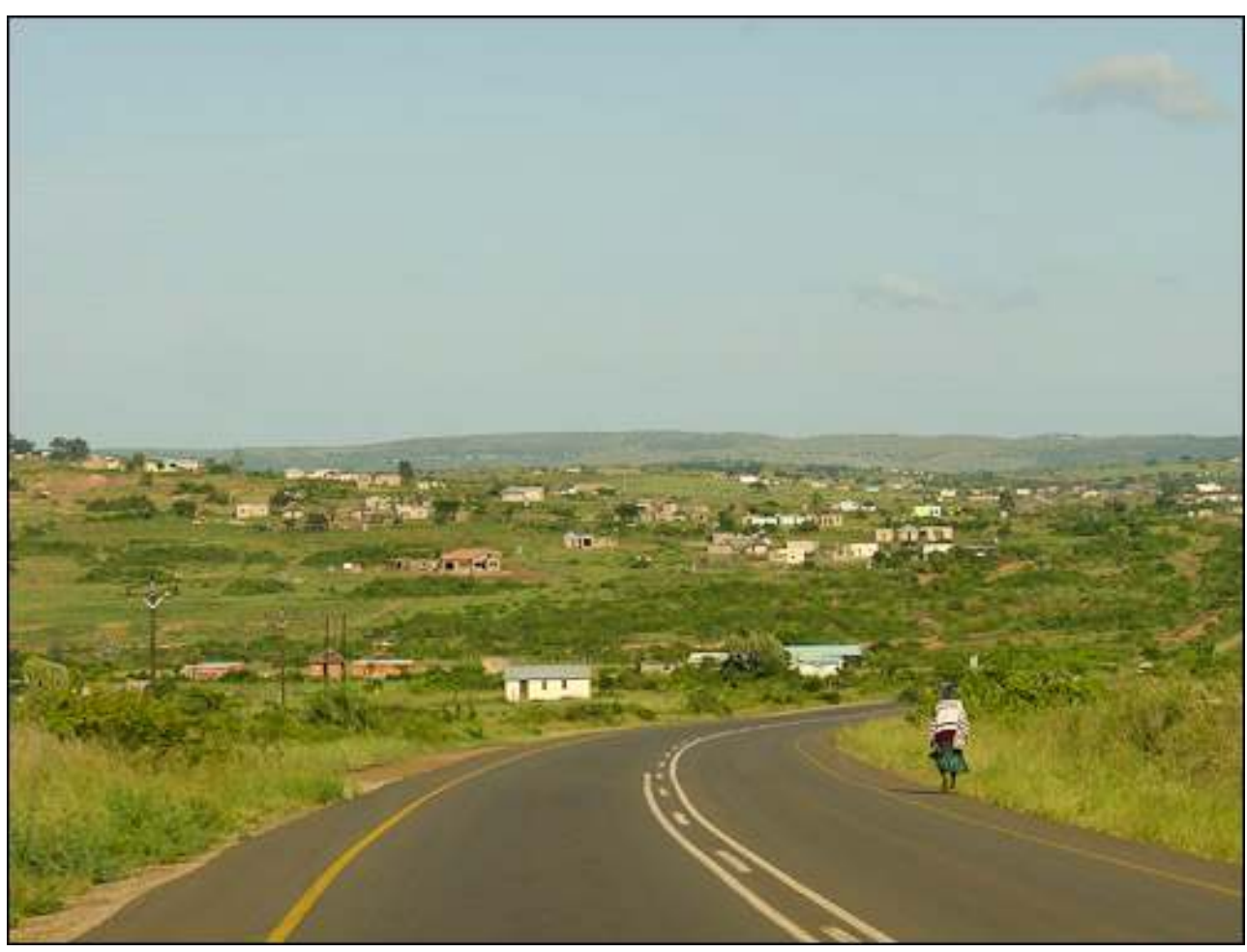

Sur la R618 menant à l'entrée Memorial Gate, un habitat semi-dispersé sous régime coutumier se dévoile au regard, autour de l'isolat naturel qu'est HIP.

Auteur : Fabrice Folio.

Dépassant cette vision traditionnelle, le EKZNW se veut dorénavant actif dans l'établissement d'une relation dynamique avec les communautés rurales. L'intérêt est de transformer les attitudes neutres ou négatives passées en comportements 
mutuellement bénéfiques. Par conséquent, la participation communautaire fait partie intégrante des politiques de l'organisme, et ce en raison de la pauvreté locale (le taux pour la province est de 54,3\% contre 49,8\% en Afrique du Sud, Provide, 2005). Depuis le $1^{\text {er }}$ février 1998, chaque visiteur, se voit ponctionner un tiers de son droit d'entrée dans le Parc. L'argent, accumulé dans un fond (community trust), sert à développer des projets communautaires environnants. Les déboursements sont effectués au travers des Local Boards. Depuis 1997 (loi $n^{\circ} 9$ du KwaZulu-Natal Nature Management, préconisant un système de cogestion, Rey, 2003), ces conseils locaux, sans précédent en Afrique du Sud (HIP faisait en effet partie des projets pilotes), tentent de réunir, dans une optique de dialogue, acteurs du Parc, chefs coutumiers, agriculteurs et organes touristiques et de commerces nommés par la province.

En juin 1999 au Camp HillTop, un forum de discussion a permis de détailler les options de partage de la taxe avec les chefs traditionnels. Le département de l'aménagement du KZN Wildlife proposa une variété d'options (écoles, cliniques, crèches, projets d'approvisionnement en eau ou bourses d'études). On demanda aux chefs d'identifier des projets pouvant se réaliser autour de la réserve. Après une heure de délibération en interne, les amaKhosi se mirent unanimement d'accord pour investir 730737 rands de la taxe communautaire dans la création d'un hébergement touristique situé à l'intérieur du Parc ${ }^{16}$. Qu'une communauté ait volontairement choisi d'investir dans une aire protégée, marque sans aucun doute un temps fort de la conservation naturelle nationale. Cette démarche équivaut à un changement de pensée des populations concernant leur environnement. D'une part, elles reconnaissent les bénéfices - à moyen et long terme - suscités par le secteur touristique en termes d'emplois et de recettes. D'autre part, l'initiative insuffle une nouvelle confiance à l'endroit de la protection naturelle et légitime l'utilisation de terres à de telles fins. On soutiendra que c'est bien la logique marchande de la conservation qui l'emporte in fine, ces initiatives participatives se tournant résolument vers le domaine économique (Rodary, 1998). 
Illustration 7a - Centre artisanal Vukuzame à l'entrée Memorial de la réserve

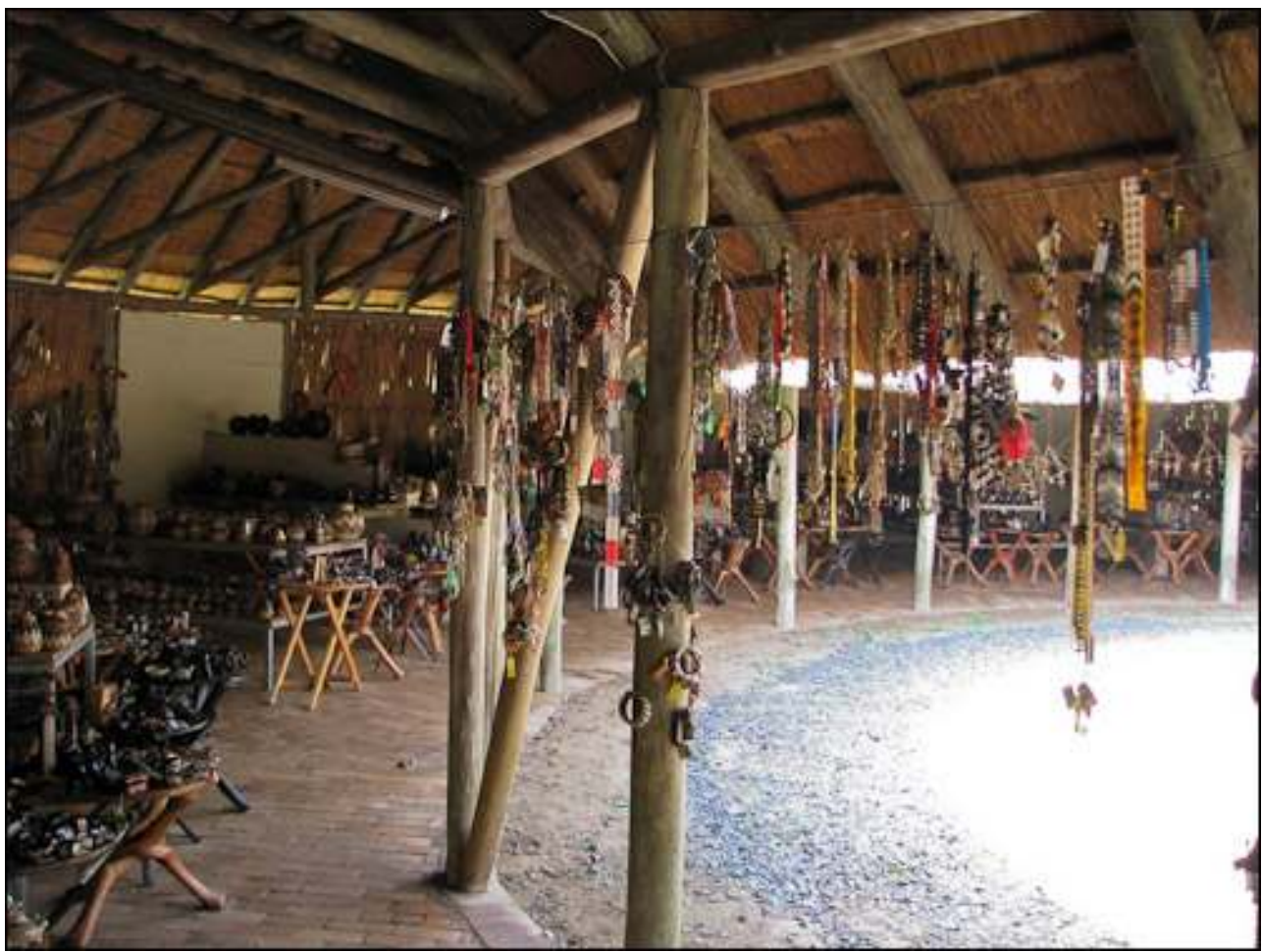

Auteur : Fabrice Folio.

Illustration 7b - Le Nswelweni Bush Lodge en construction en 2009

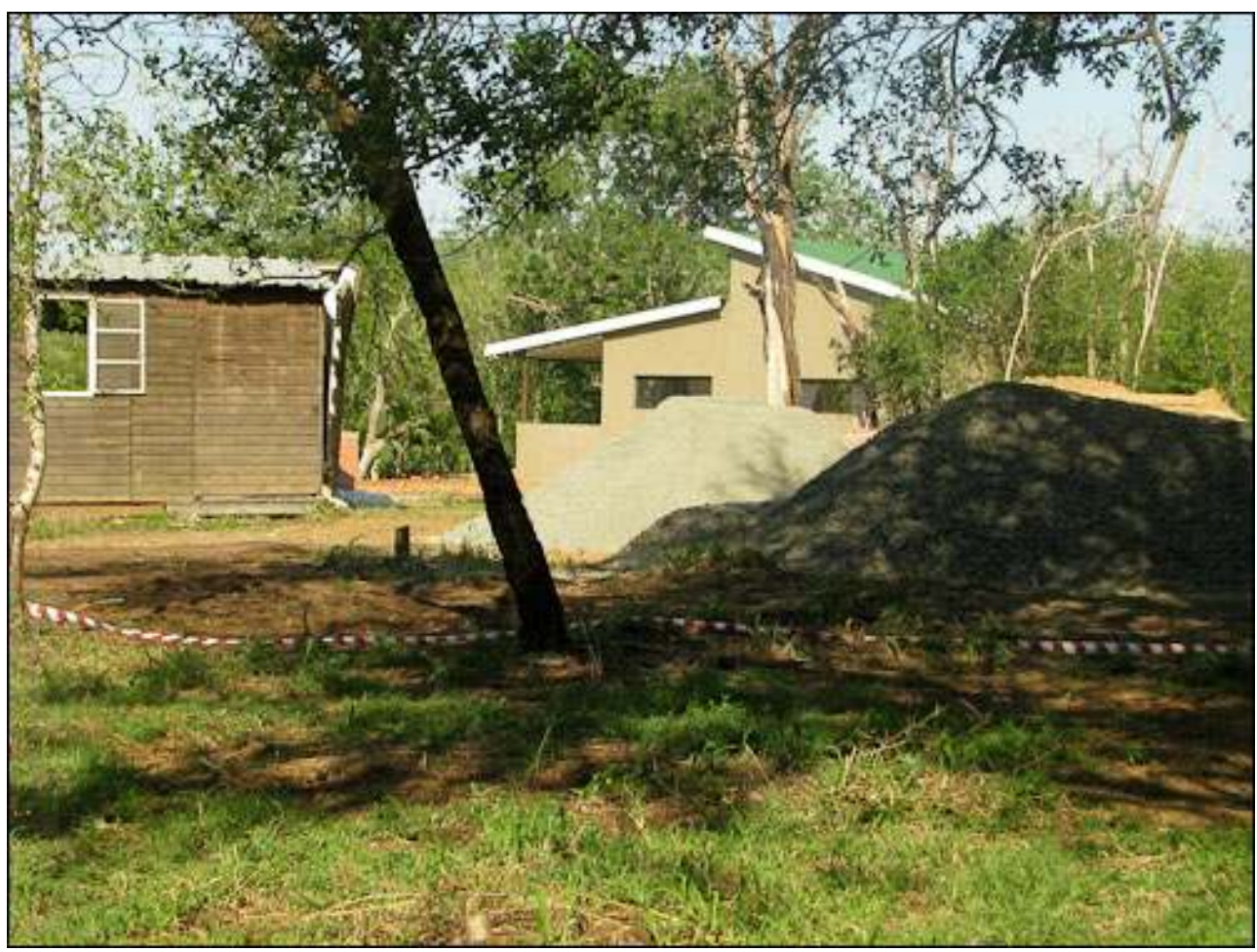

Auteur : Fabrice Folio. 


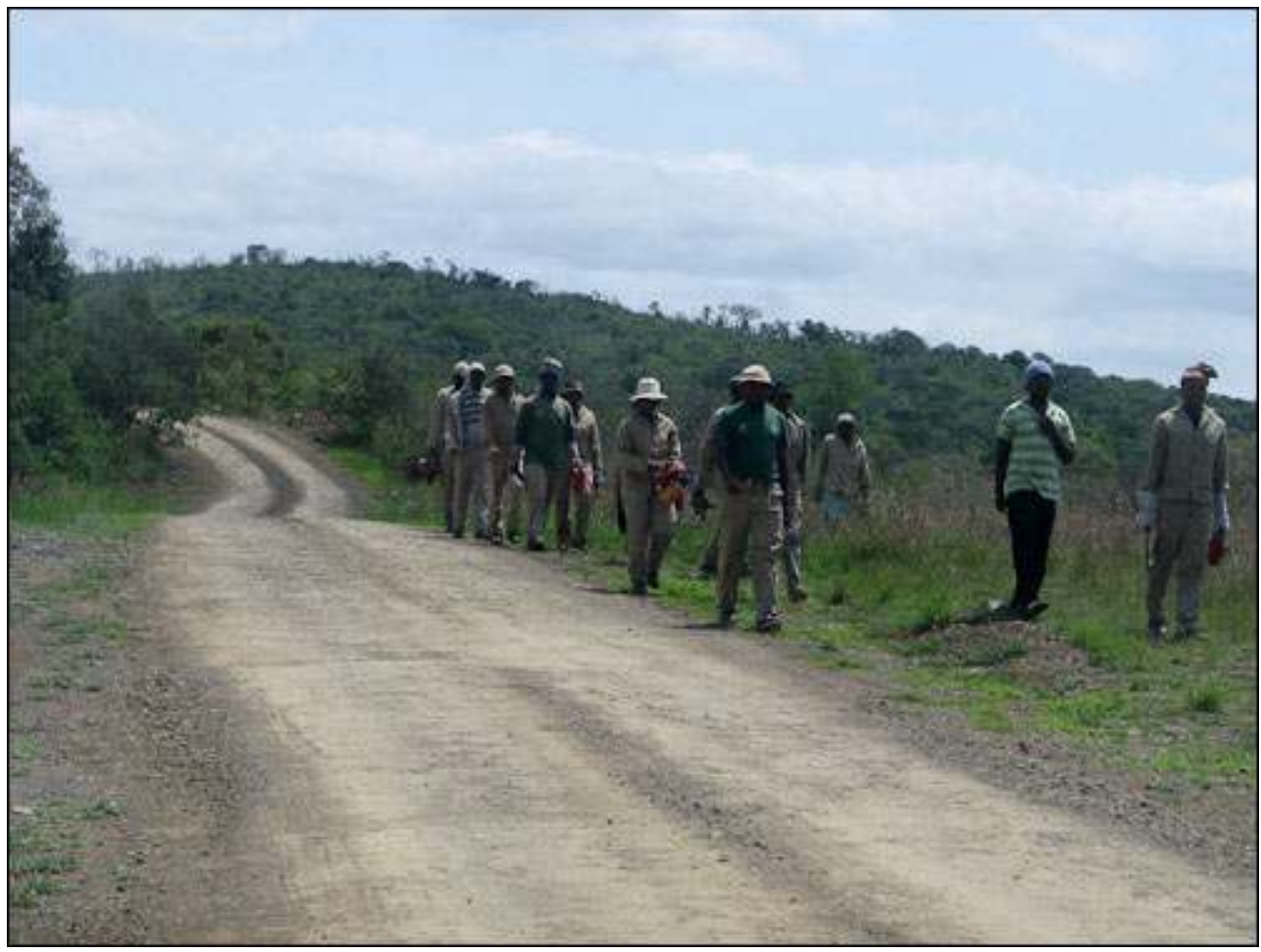

Auteur : Fabrice Folio.

\section{Une équation résolue à HIP ? Une approche écotouristique intéressante mais ambiguë, sur fond de vifs enjeux territoriaux}

Au total, si on peut parler, dans une certaine mesure, d'une réalité écotouristique à HIP, cela ne signifie pas qu'elle soit en tout point exemplaire. Il existe quelques dérives causées par l'activité et des insuffisances demeurent (bien que le Parc ne puisse régler, à lui seul, tous les problèmes de la région). Plus largement, c'est la question de la sanctuarisation même et celle des interactions et contradictions entre acteurs qui peuvent être reposées.

Si au plan environnemental et économique l'équilibre semble de mise, au titre de la redistribution environnante, des limites existent. Les possibilités d'emplois dans le Parc restent réduites et les salaires bas et/ou saisonniers (430 rands mensuels en moyenne ou $40 €)$ : en raison d'une faible qualification, les emplois proposés sont souvent liés à l'assistance générale (femmes de chambre, agents d'entretien...). Quant aux défis socioéconomiques environnants, ils restent immenses. Par ailleurs, toutes les communautés proches ne sont pas égales dans leur importance. Après observation, ces aires villageoises comportent des niveaux d'équipements et d'infrastructures disparates. Sans surprise, cela est surtout du à des motifs externes (proximité d'axes routiers fréquentés, de localités comme Mtubatuba). Mais en tout état de cause, doivent être relativisés les effets homogénéisants de la présence du Parc parmi les communautés attenantes. En revanche, il a été avancé par nos interlocuteurs une pression démographique plus forte dans la région, causée justement par une poussée migratoire liée aux espoirs de profit dégagés par les sites de HIP et de St-Lucia. Pour finir, du côté de l'équipe du Parc, on soutient que l'équilibre écotouristique reste fragile. La chance 
de HIP est en fait de pouvoir dégager des recettes supplémentaires pour la conservation : en plus des subventions gouvernementales et des dépenses des visiteurs, ce sont surtout les mises aux enchères du surplus d'animaux (à des Parcs qui en sont dépourvus ou à des prestataires privés) qui lui assurent des devises substantielles (la chasse étant ici bannie): HIP glane chaque année, lors de ses Game Auctions tenus aujourd'hui au Sibaya Casino à Durban, plus de R10 millions; son Capture Centre a été érigé en modèle. L'hébergement ( $49 \%)$ et la vente d'animaux ( $28 \%)$ occupent à ce titre les premiers postes des revenus annuels. N'y étant que peu indexée, la taxe communautaire ne représente elle que R2 millions du budget total.

Un autre sujet de réflexion et de débat porte sur la nature même de la sanctuarisation écologique et de ses rapports avec les espaces environnants. Quels que soient les types d'interactions existants (et ils ont ici le mérite d'exister), la création de réserves naturelles s'est par le passé opérée dans un écosystème anthropisé, même si nous est à présent offert, comme produit d'appel, une Afrique naturelle figée, dépourvue d'hommes. Le modèle européanocentré de la nature, d'inspiration coloniale, s'est imposé au détriment des populations locales. A l'heure actuelle, une approche conservationniste, dans l'air du temps, fait la part belle au tourisme - en qualité de moteur de développement d'un pays émergent -. Mais les écotouristes actuels, empreints d'un tel système de représentations, ne s'apparentent-ils pas, par certains aspects, aux visiteurs britanniques du $19^{\mathrm{e}}$ siècle (Ramutsindela, 2004) ? Certes, pour les besoins de la cause, la conservation met clairement en avant la participation communautaire, soit un revirement eu égard au passé. Toutefois, au-delà de la question de la généralisation de cette communauté en tant que partenaire idéalisé instrumentalisé ? - considéré aussi comme structure sociale homogène (un point dénoncé par Agrawal et Gibbson, 1999), il reste qu'une forme de territorialisation prend effet en cet endroit, lorsque des visiteurs extérieurs (internationaux ou sud-africains plutôt aisés) s'approprient un espace clos autour duquel vivent des populations du Sud (qu'ils aperçoivent en accédant au Parc dans une forme "d'effet tunnel écologique »). Cette « communauté » n'est finalement qu'assez peu associée à l'activité touristique et les relations entre ces acteurs - qui ne s'ignorent pas - demeurent de la sorte réduites. L'effet en est accentué par l'héritage de la politique d'apartheid, qui a consacré au plan spatial deux territoires, réunis en $1994^{17}$.

On soutiendra que le modèle effectif à HIP est loin de ne comporter que des points négatifs. La sensibilisation, les instances de discussion et l'ébauche de participation sont autant d'aspects constructifs et symbiotiques. Pourtant il apparaîtrait judicieux de davantage "désenclaver " touristes et villageois adjacents et faire en sorte que ces acteurs puissent interagir. La finalité en serait une meilleure compréhension du milieu local et de ses enjeux. A l'observation du discours écotouristique, on est par exemple frappé de voir que l'interprétation du patrimoine culturel demeure localement ténue. Or, celui-ci gagnerait à être aisément valorisé dans un pays comme l'Afrique du Sud. Evidemment, le risque à ce stade, comme le rappellent fort à propos F. Giraut, S. Guyot et M. Houssay-Holzschuch (2004), est qu'une potentielle participation à la valorisation de la culture locale ne joue la carte d'une autochtonisation déplacée, digérant un conservatisme exotique fait d'ordre social hiérarchisé et statique (l'exemple type étant les Cultural villages surfaits, tels "Shakaland" près d'Eshowe, dans cette même province). Dans l'idéal, non seulement les visiteurs pourraient bénéficier d'une 
interprétation du patrimoine naturel et culturel de la zone, mais aussi les populations locales seraient mieux intégrées dans l'activité touristique du Zululand ${ }^{18}$.

Un ultime sujet de réflexion a trait aux logiques d'acteurs sur le terrain, en rapport avec les enjeux en cours. Elles jettent finalement un éclairage très pragmatique sur les relations entre le Parc et les populations riveraines. Le EKZNW doit faire face à l'heure actuelle à de nombreuses revendications foncières ou land claims; celles-ci renvoient aux expropriations historiques. A l'image du pays, ces revendications sont vives, y compris concernant les parcs naturels (on cite souvent les exemples de Ndumo ou de Tembe dans la province). Au total au KwaZulu-Natal, 407 land claims ont été lancées dont 33 sur des terres étatiques. L'intérêt du EKZNW n'est pas de rétrocéder ces aires territoriales mais bien d'arriver, par la concertation, à faire bénéficier les communautés revendicatrices et spoliées des opportunités touristiques et de conservation. Les populations locales poussent évidemment cet aspect à leur avantage, dés lors que le panel possible devient important : partage des revenus dégagés par les entrées des aires protégées, activités commerciales associées, accès aux sites ancestraux ou aux ressources naturelles. On saisit alors que confrontées à de telles demandes légitimes, qui plus est instrumentalisées politiquement, les autorités des Parcs - et HIP ne fait pas exception - ne peuvent que mettre à l'ordre du jour ces logiques de conciliation et d'association communautaire. A terme, de nouveaux espaces protégés en cogestion communautaire pourraient même voir le jour. Ainsi, certaines land claims résolues ont déjà vu s'établir des réserves privées (comme celle de Somkhanda sur l'initiative de l'autorité tribale de Gumbi près de Pongola); une land claim peut donc produire du tourisme de nature voire de l'écotourisme. Dans la zone d'étude, le Hluhluwe-Imfolozi corridor fait à l'heure actuelle l'objet d'une revendication (Tribune, 2008). D'une conservation avec les populations, on tendrait de cette manière vers une conservation par les populations (Compagnon et Constantin, 2000). Les Local Boards, malgré leurs maigres moyens et capacités opérationnelles, accordent de la sorte une place de choix aux autorités tribales très connotées historiquement et politiquement. Cela a pour incidence de court-circuiter les formes de gouvernement local (Luckett, Mkhize, Potter, 2003) et de complexifier le jeu politique interne. In fine et écornant leur légitimité, les nouvelles autorités municipales (local et district municipalities) ne sont pas tant associées que cela aux divers enjeux territoriaux : elles doivent composer avec ces formes héritées d'autorités locales consolidées.

Avec la médiatisation du concept écotouristique au plan international, on prend aujourd'hui conscience de la gageure qu'il incarne, parallèlement, dans les pays en développement; cela, sans oublier les impacts potentiels de cette activité, au-delà même de ce qui devenu un label (Keyser, 2002). L'exemple du parc animalier de Hluhluwe-Imfolozi au KwaZulu-Natal insiste sur la nécessité de saisir la complexité des acteurs et des niveaux d'échelle dans la lecture d'une dynamique écotouristique de prime abord justifiée.

Par delà les discours qui semblent sur place imprimer une certaine cohérence dans le profil d'ensemble (en mettant bout à bout les initiatives sociales, sous l'ombrelle économique et environnementale), des limites et fragilités perdurent. Si HIP fait état de démarches intéressantes, aidées en cela par une gestion rigoureuse et ancienne, les insuffisances restent aussi liées à un contexte africain et sud-africain hérité. La valorisation culturelle est ainsi localement minorée, alors que le tourisme peut constituer un instrument de mise en valeur ou de revivification des pratiques. De plus, 
alors que des projets participatifs et de redistribution existent, ce sont surtout les recettes émanant du Parc qui lui autorisent une certaine marge de manœuvre, à l'intérieur d'un pays dont il faut encore rappeler qu'il demeure très impliqué dans les démarches d'écotourisme (à l'instar de l'Australie, Laurent, 2003) ${ }^{19}$. Mais considérant ici l'étendue des défis sociaux, l'arrière-plan historique d'expropriation et enfin les risques de fuites de capitaux dans la contribution écotouristique à l'économie locale (Rogerson, Visser, 2004), il convient de porter à l'actif de HIP l'implication progressive des populations environnantes dans les instances de discussion, ainsi que la ventilation des taxes communautaires dans des projets concrets. En cela, la gestion du Parc, d'une visée écotouristique, tend un peu plus vers une forme de tourisme communautaire (qui accroit l'engagement local). Pour autant, cette forme de revalorisation des acteurs locaux, de "globalisation par le bas» (Hunter, 1995), ne doit pas faire oublier des décisions et choix impulsés/influencés par le haut. De surcroît, les conséquences ne s'orientent-elles pas sur le rôle majoré que prennent ces partenaires et le risque de politisation des enjeux? La logique écotouristique contemporaine, en faisant le jeu d'une Afrique imagée et fantasmée, mobilisant l'échelon traditionnel historique (à défaut des nouvelles municipalités démocratiques), ne resterait-elle pas, au final, effective mais par certains aspects conservatrice?

\section{BIBLIOGRAPHIE}

Adams J.-S., Macshane T.-O., 1996. The Myth of Wild Africa. Conservation without illusions. Berkeley, University of California Press.

Agrawal A., Gibson C.-C., 1999. Enchantment and Disenchantment: The Role of Community in Natural Ressource Conservation; World Development, 27 (4), p. 629-649.

Amakhosi put their faith in conservation, 1999. IOL, 19 juillet.

Aylward B., Lutz E., 2004. Nature, Tourism, Conservation and Development in KwaZulu-Natal, SA. Ed. Durban, The World Bank, $450 \mathrm{p}$.

Belaidi N., 2008. Le Great Limpopo Transfrontier Park. EchoGéo, Numéro 7 | 2008, mis en ligne le 16 octobre 2008. URL : http://echogeo.revues.org/index8523.html .

Blamey R.-K., 2001. Principles of Ecotourism. In The Encyclopedia of Ecotourisme, Ed. Oxon, UK, New York, p. 5-22.

Brave park ranger gave up his life for us, 2004. Lyse Comins in The Mercury, $1^{\mathrm{er}}$ avril.

Brooks S., 2001. Changing Nature: A Critical Historical Geography of the Umfolozi and Hluhluwe Game Reserves, Zululand, 1887 to 1947. PhD Thesis, Dpt of Geography, Queens University (Canada).

Brunel S., 2005. Quel tourisme en Afrique ? In L'Afrique dans la mondialisation, documentation géographique, $\mathrm{n}^{\circ} 8048$.

Brunel S., 2004. L'Afrique, un continent en réserve de développement. Bréal, 239 p. 
Calas B., 2003. Quel est le véritable patrimoine des parcs animaliers est-africains ? In Patrimoine et développement dans les pays tropicaux, Pessac, DYMSET (Espaces Tropicaux, $\mathrm{n}^{\circ} 18$ ), $\mathrm{p} ; 333-342$.

Carron M., 2005. Gestion des Réserves Fauniques de la République Sud-Africaine : les limites du modèle réduit et les voies d'amélioration. PTM-441 Pathologie de la Faune et de l'Environnement, $17 \mathrm{p}$.

Charlton Perkins W., 2002. Hluhluwe Imfolozi Park. In Great Game Parks of Africa, Ed. Struik Publisher, Pretoria, p. 55-75.

Christie I.-T., Crompton, D.-E., 2001. Tourism in Africa. Africa Working Paper Series, $\mathrm{n}^{\circ} 12$, Banque Mondiale (http://www.worldbank.org/afr/wps/index.htm).

Compagnon D., Constantin F., (éd), 2000. Administrer l'environnement en Afrique. Paris/Nairobi, Karthala/IFRA, 497 p.

Derroisne A., 2005. L'écotourisme dans une réserve naturelle d'Afrique du Sud: le cas du Parc faunique de Hluhluwe/Imfolozi, Mémoire de Master de géographie, sous la direction de Folio F., CREGUR, Université de La Réunion, 140 p.

Ellis S., 1992. Défense d'y voir : la politisation de la protection de la nature. Politique Africaine, $\mathrm{n}^{\circ}$ 48, L'Afrique du Sud, la nouvelle donne, p. 7-21 (http://www.politique-africaine.com/ numeros/pdf/048007.pdf).

EKZNW, Celebrates 10 Years of Democracy, 2004. Ezemvelo KZN Wildlife, 24 p.

Gervais-Lambony P., 2009. L'Afrique du Sud. Collection Idées reçues, Le Cavalier Bleu, 127 p. Giraut F., Guyot S., Houssay-Holzschuch, M., 2004. Les aires protégées dans les recompositions territoriales africaines. L'Information Géographique, vol. 68, décembre, p. 340-368.

Guyot S., 2006 (a). Rivages zoulous. L'environnement au service du politique en Afrique du Sud. IRD et Karthala, 250 p.

Guyot S., 2006 (b).Les parcs naturels d'Afrique australe : d'autres territoires de conflits. Géoconfluences, le 28 février 2006, http://geoconfluences.ens-lsh.fr/doc/etpays/Afsubsah/ AfsubsahScient2.htm

Guyot S., 2006 (c). Géopolitique des Parcs (trans)frontaliers en Afrique Australe. Les Cahiers d'Outre Mer, n 234 2006/2, p. 215-232.

Guyot S., 2004. Derrière le masque de l'écotourisme, le politique : conservation et discrimination territoriale en Afrique du Sud. Tiers-Monde, t. XLV, n 178, p. 341-363.

Honey M., 1999. Ecotourism and sustainable development: who owns paradise? Washington, Island Press, $405 \mathrm{p}$.

Hunter A., 1995. Globalisation from below? Promises and perils of the new internationalism. In Social Policy, $\mathrm{n}^{\circ} 25$.

I thought I was finished, says rhino victim, 2003-b. Graeme Hosken in The Mercury, 9 mai.

Keyser H., 2002. Tourism and Development. Ed. Oxford University Press, Cape Town, 200 p.

Koch E., 2001. Tales of white elephants - ecotourism in South Africa. Brief Article UNESCO Courier.

KZN Wildlife's revenue goes through the roof, 2003-a. T. Carnie in The Mercury, 8 aout.

Lamy-Giner M.-A., Guébourg J.-L., 2005. Le tourisme international en Afrique du Sud. Cybergeo, Espace, Société, Territoire, article 331, mis en ligne le 12 décembre 2005.

Laurent A., 2003. Les acteurs entreprenants du Sud dans le développement du tourisme responsable. L'actualité des services aux entreprises, $\mathrm{n}^{\circ}$ 6, DGCID (MAE), p. 5-9. 
Lubbe C. Tourism Management in South Africa. Ed. Berendier, Cape Town, 170 p.

Luckett S., Mkhize K., Potter D., 2003. The experience of Local Boards in KwaZulu-Natal, South Africa. Parks, vol. 13, n 1, p. 6-15.

Mackenzie J.-M., 1988. The empire of Nature. Hunting, Conservation and British imperialism. Manchester Univ. Press (coll. Studies in Imperialism).

Matlou P., 2001. The Potential of Ecotourism Development \& its Partnership with Spatial Development Inititiatives. Séminaire Planning, Development and Management of Ecotourism in Africa, Maputo, Mozambique, 5 - 6 mars 2001, 12 p.

Ndebele N. Game lodges and leisure colonialists. In H. Judin et I. Vladislavic (éd.), Blank Architecture, apartheid and after, Capte Town, David Philip Publishers, p. 119-123.

Organisation mondiale du tourisme (OMT), 2003. Le tourisme et la réduction de la pauvreté. L'actualité des services aux entreprises, $\mathrm{n}^{\circ}$ 6, DGCID (MAE), p. 10-13.

OMT, 2007. Faits saillants du tourisme. Edition 2007, 12 p.

Pourtier R., 2001. Afriques noires. Hachette, 255 p.

PROVIDE, 2005. A profile of KwaZulu-Natal : Demographics, poverty, inequality and unemployment. Background Paper 2005 :1(5), Provide Project, Elsenburg. http:// www.elsenburg.com/provide/documents/BP2005_1_5 \%20Demographics \%20KZ.pdf

R15m allocated to fight alien plants, 2003. T. Carnie in The Natal Mercury, 21 février.

Ramutsindela M., 2004. Parks and people in postcolonial societies: experiences in Southern Africa. Dordrecht, Kluwer Academic Publishers, 192 p.

Rey B., 2003. Une nouvelle territorialité pour une nouvelle conservation de la nature au KwaZuluNatal ? Actes bilingues des rencontres franco sud-africaines de l'innovation territoriale, laboratoire Territoire (UMR PACTE, Université Joseph Fourier), édition de l'IRD dans la collection «Colloques et séminaires » (2003) ; 5 p. (http://www.pacte.cnrs.fr/IMG/ pdf_30_ReyConservation_KZN.pdf).

Rodary E., 1998. De la conservation participative à la promotion de l'économie libérale. Les politiques de conservation de la faune en Zambie et au Zimbabwe. In Politique Africaine $n^{\circ} 72, p$ 113-129 (http://www.politique-africaine.com/numeros/pdf/072113.pdf).

Rogerson M., Visser G., 2004. Tourism and Development Issues in Contemporary South Africa. Institute of South Africa, $156 \mathrm{p}$.

Rossi G. L'ingérence écologique. Environnement et développement rural du Nord au Sud. Paris, CNRS Editions.

Thresher P., 1981. The economics of a lion; Unasylva, vol 33, n 134, p. 34-36.

Traditional leader fights for nature, 2008. M. Ryan in Tribune, 8 juin.

Volvey A., (dir.), 2005. Le Parc National. In L'Afrique, Atlante, p. 204-206.

Wackermann G., 2004. Effets turbulents de la mondialisation de l'économie récréative. In L'Afrique en questions, A.-M., Frérot (dir.), Ellipses, p. 37-41.

Weisrock A., 2004. Peut-on sauver la nature en Afrique ? In L'Afrique en dissertations corrigées et dossiers, G. Wackermann (dir.), Ellipses, p. 105-113.

Wilkie D.-S., 1999. Ecotourisme en Afrique centrale, tourisme et conservation ou le mariage de la carpe et du lapin? In Canopée, $\mathrm{n}^{\circ} 13$. 


\section{NOTES}

1. Aires protégées abritant les grands fauves, peintures rupestres san, villages traditionnels bantous, vignobles ou espaces naturels (canyon, cap, plage, lagune, montagne...) et urbains (mall, township) fondent ses atouts.

2. Le plus connu est le Great Limpopo Transfrontier Park depuis 2006, entre le Parc Kruger et ceux de Gaza et de Gonarezhou au Mozambique et au Zimbabwe (même si le premier reste celui de Kgalagadi inauguré en 1999).

3. Au Kenya, le tourisme reste un grand pourvoyeur de monnaie étrangère (Honey, 1999). En 1996, il représentait déjà une forte contribution au PNB, avec 474 millions de US \$ de revenus (Volvey, 2005). Des études dans le Parc d'Amboseli ont montré que chaque lion «vaut » environ 515000 dollars en devises (Thresher, 1981).

4. Bien qu'à Johannesburg, dix ans plus tard, des contradictions et blocages persisteront lors du second sommet de la Terre, du fait d'objectifs trop ambitieux et d'une pluralité d'acteurs et de stratégies pas forcément compatibles.

5. Sont ici fréquemment avancés les exemples camerounais (village d'Ebodje), sénégalais (campement intégré de Casamance) ou encore malgache (Parc national de Ramonafan).

6. Les expériences africaines les plus poussées qui nous sont rapportées sont les projets CAMPFIRE et ADMADE au Zimbabwe et en Zambie ou la restitution/optimisation foncière des Makuleke au Parc Kruger.

7. La recherche sur place a d'abord consisté en une recherche bibliographique à l'Université du KwaZulu-Natal à Durban, la capitale économique de la province. La plupart des bureaux administratifs, du siège des organismes environnementaux étant situés ici ou à Pietermaritzburg (la capitale administrative provinciale), des entretiens ont en parallèle été conduits auprès de membres du Ezemvelo KZN Wildlife (l'organisme provincial de conservation de la nature). Par la suite, un travail de terrain (Desrroine 2005, Folio 2009) a été réalisé sur le site. En plus des visites du Parc, des discussions ont été menées auprès de personnes locales, notamment des membres des communautés résidentes à proximité de la réserve. Afin de jauger la fréquentation touristique de HIP, un questionnaire a finalement été administré à une centaine de visiteurs aux trois entrées du Parc.

8. Le KwaZulu-Natal est la plus petite mais aussi la plus peuplée des provinces d'Afrique du Sud. Terre de rencontre des identités zouloue, anglaise et indienne, il s'ouvre sur l'océan Indien au nord-est du pays. La province bénéficie d'un climat subtropical océanique humide, alors que $60 \%$ des terres sud-africaines reçoivent moins de $700 \mathrm{~mm}$ de précipitations. La topographie de HIP varie de 60-80 mètres d'altitude en lit de rivières à 540-600 $\mathrm{m}$ sur les chaînes de collines. Les cours d'eaux qui le sillonnent sont notamment la rivière Hluhluwe au nord-est et les larges et peu profonds Black et White Mfolozi au sud. Les précipitations moyennes annuelles sont de $950 \mathrm{~mm}$ à Hluhluwe (Camp HillTop) et de $650 \mathrm{~mm}$ à Imfolozi (Camp Mpila).

9. Dont l'Evelea, le Tamboti et la Marula qui sont des espèces d'arbres exotiques du Zululand (un des 11 districts du KwaZulu-Natal); HIP se targue de surcroît d'abriter le typique thorveld (buisson épineux), alors que ses paysages de savanes sont surtout caractérisés par l'emblématique acacia des réserves naturelles africaines.

10. Le chauffeur est un guide qui promène ses clients (jusqu'à dix par véhicule) sur les sentiers qu'il connaît bien. Il leur fait profiter d'informations précises sur les espèces qu'ils rencontrent, tout autant que d'anecdotes personnelles. Les tours peuvent se réaliser sur une demi-journée ou sur 12 heures et les repas y sont inclus. Les clients peuvent de surcroît profiter de tours nocturnes, qui sont une opportunité de voir d'autres espèces animales (Léopard...) éclairées au spot lumineux.

11. Menés en basse saison touristique sud-africaine, les sondés dégageaient une légère majorité d'étrangers (Européens - Allemands et Anglais - suivis de Nord-américains), le KwaZulu-Natal 
étant parallèlement une province où un fort tourisme domestique prend place (du à sa proximité avec les hautes terres du Gauteng).

12. A ce stade, les informations proviennent pour beaucoup des entretiens menés auprès du Tourism KwaZulu-Natal (Mr W. Tifflin en 2009) et surtout auprès d'interlocuteurs du EKZNW : Mr. Redd, Natural conservator, Mr. Hlabisa et Gcumisa du Group Conservation Partnerships (2005) et Mrs. Zimi (Communication, 2009).

13. Cette contrée est en effet connue pour sa culture zulu et ses champs de bataille anglo-boerzoulou ou Battlefields (Blood River, Isandhlwana...). Surtout, se nichent ici de nombreux espaces protégés (Ithala, uMkuze et la réserve privée de Phinda, illustration 3); le plus illustre est le Isimangaliso Wetlands Park (auparavant Greater St Lucia), vaste site littoral classé patrimoine mondial de l'humanité et situé à 30-40 km de HIP à vol d'oiseau.

14. De taille modérée (capacité totale de 325 lits), ils sont construits avec des matériaux naturels tels que le bois et le chaume (modèle de l'ecolodge). L'architecture utilisée, en rondavels ou huttes, s'est aussi « autochtonisée ». S'ils n'ont pas d'équipements pour utiliser les énergies naturelles, la plupart des hébergements récoltent cependant l'eau de pluie pour l'arrosage ou les travaux d'entretien. Ils gèrent aussi leur propre assainissement.

15. Par exemple, les populations accèdent gratuitement et sous contrôle à certaines sections sélectionnées de la réserve. Elles peuvent ici se servir en surplus de ressources produites par le milieu, en prélevant du bois pour le feu, des roseaux ou encore du chaume (leur valeur annuelle est estimée à R500 000).

16. Soit l'équivalent de plus de $68000 €$. Les négociations furent âpres; quelques-uns d'entre eux auraient au départ préféré un placement dans une corporation financière (IOL, 1999). Les chefs arguèrent finalement que diviser la somme aurait été injuste compte tenu des différences de taille entre les dix autorités tribales et de leurs effectifs humains hétérogènes. Qui plus est, certains d'entre eux avaient construit des huttes traditionnelles en forme de ruche ; ils avaient déjà pu noter leur relative popularité auprès des touristes internationaux...

17. Avant de pénétrer à HIP, le visiteur traversera ainsi des parcelles d'agriculture de plantation (ex-Afrique du Sud blanche), avant de changer de monde et de longer un habitat zoulou semi dispersé (ex-bantoustan Kwazulu). Une certaine Afrique traditionnelle (après celle développée), au passé chargé, lui est ainsi proposée (à son insu mais ne le laissant guère indifférent), avant celle de la nature sauvage. Le zonage est perpétué.

18. Parmi les initiatives à valoriser, on pourrait par exemple inciter la mise en place d'ateliers culturels sous forme associative, dont les possibilités seraient, entres autres, de raconter l'historique pré et post colonial du Parc (logique mémorielle). Favoriser les expositions artistiques, animer des démonstrations des multiples savoir-faire artisanaux et composer des visites guidées de sites patrimoniaux sont d'autres possibilités. La dynamique pourrait prendre effet dans les aires environnantes comme à des points stratégiques situés dans la réserve. L'initiative du Centenary Center, ouvert en 1995 à Imfolozi, structure audio-visuelle à disposition du public et des écoles de la région, liée à la conservation et aux captures, est à ce titre une entreprise intéressante.

19. Rappelons que l'Afrique du Sud couvre depuis longtemps $70 \%$ de ses coûts de gestion d'aires protégées avec les revenus générés dans ses Parcs et réserves (Wilkie, 1999). Le pays cumule richesse écologique, bon niveau de prestations et relative notoriété. Or, l'on sait que, trop souvent, en d'autres cieux, les recettes de l'écotourisme restent modestes et ne suffisent pas à assurer des normes élevées de gestion de l'environnement. 


\section{RÉSUMÉS}

Comptant parmi les plus vieilles réserves naturelles d'Afrique australe, l'aire protégée de Hluhluwe-Imfolozi Parc (HIP) se situe dans la province du KwaZulu-Natal en Afrique du Sud. Elle est aujourd'hui représentative d'une Afrique du Sud sauvage et ouverte aux visiteurs, dans un pays ayant fait une priorité des axes de durabilité/responsabilité touristique (sur la base d'un fort potentiel, de prestations de qualité et de l'orientation politique). En s'engageant dans la voie écotouristique, HIP est le cadre d'initiatives, en la matière, qualifiées de novatrices lors de leur établissement. Toutefois, le constat d'ensemble n'est pas exempt de fragilités, qu'il convient aussi de rapporter à d'importants défis hérités. Au-delà des résultats encourageants et des insuffisances notables, la politique du Parc, derrière la flexibilité touristique et la progression de l'échelon communautaire limitrophe, reste pour le moins ambiguë, dans ses aspects territoriaux comme dans l'implication des acteurs.

As one of the oldest game reserves in austral Africa, the Hluhluwe-Imfolozi Park (HIP), set in the heart of the KwaZulu-Natal province, has become a south-african example as a conservation sanctuary involved in ecotourism practices. Its rich biodiversity has paved the way to protection, tourism and participation initiatives during its development. Located in a country, which also gave priority to the sustainable/responsible tourism issues (in relation to its rich potential, level of services as well as strong governmental policy), the results are not free from several fragilities and insufficiencies. One also has to relate these facts to the inherited national socio-economical stakes. Finally, the particularity of HIP led in a mixed of seducing and, in such way, ambiguous ecotourism reality, putting together a strong and old provincial conservation programme, a diversified and adaptable tourism activity as well as a gradual involvement from the nearby tribal authorities in the Park policy.

\section{INDEX}

Mots-clés : Afrique du Sud, autorité tribale, biodiversité, conservation, écotourisme, Ezemvelo KZN Wildlife, Hluhluwe-Imfolozi Park, KwaZulu-Natal, participation

Keywords : biodiversity, conservation, ecotourism, Ezemvelo KZN Wildlife, Hluhluwe-Imfolozi Park, KwaZulu-Natal, participation, South Africa, tribal authority

\section{AUTEURS}

\section{FABRICE FOLIO}

Fabrice Folio (fabrice-jm.folio@univ-reunion.fr) est maître de conférences au département de géographie et membre du Centre de Recherches et d'Etudes en Géographie(CREGUR), Université de La Réunion. Ses dernières publications :

Folio F., 2009. Réalités et singularités du tourisme réunionnais : entre utopie et motifs d'espoir. In Les Cahiers d'Outre-Mer, 245 (2009).

Folio F., 2007. Construction géohistorique du KwaZulu-Natal (Afrique du Sud). Une terre de conflits et d'enjeux à l'origine du présent cadre spatial. EspacesTemps.net, Textuel (2007). 


\section{ANAËLLE DERROISNE}

Anaëlle Derroisne est une ex-étudiante du Master Géographie-Histoire, Université de La Réunion, chargée de projet développement local, Mission Intercommunale Ouest. 